\title{
A Shared Aperture Microstrip Antenna Array with Multiple Polarisations and Near-Field Beam Steering
}

This paper was downloaded from TechRxiv (https://www.techrxiv.org).

\section{LICENSE}

CC BY-NC-SA 4.0

SUBMISSION DATE / POSTED DATE

03-01-2022 / 07-01-2022

\section{CITATION}

Ali, Haider; Afzal, Muhammad; Thalakotuna, Dushmantha; Esselle, Karu; Mukhopadhyay, Subhas (2022): A Shared Aperture Microstrip Antenna Array with Multiple Polarisations and Near-Field Beam Steering. TechRxiv. Preprint. https://doi.org/10.36227/techrxiv.17712335.v1

$\mathrm{DOI}$ 


\title{
A Shared Aperture Microstrip Antenna Array with Multiple Polarisations and Near-Field Beam Steering
}

\author{
Haider Ali*1, Muhammad U. Afzal ${ }^{2}$, Dushmantha Thalakotuna ${ }^{2}$, Karu P. Esselle ${ }^{2}$ and Subhas \\ Makhupadyay ${ }^{1}$ \\ ${ }^{1}$ School of Engineering, Macquarie University, NSW, Sydney, Australia \\ ${ }^{2}$ School of Electrical \& Data Engineering, University of Technology Sydney, NSW, Australia \\ *haider.ali@hdr.mq.edu.au
}

\begin{abstract}
In this paper we present a shared-aperture polarisation reconfigurable microstrip array designed to resonate at $11.5 \mathrm{GHz}$ with a gain bandwidth of $2 \mathrm{GHz}(\sim 17 \%)$. The polarisation reconfigurability (both linear and circular) is achieved using two orthogonal and independently-fed sub-arrays that are intertwined together on the same aperture. Each subarray is fed through one port and a feed network that distributes the power among the array elements incorporating Taylor taper distribution to minimize the sidelobe level. The array has low cross-polarisation level $(<-20 \mathrm{~dB})$ and good port isolation $(<-24$ dB). The shared aperture and absence of active switching devices provide better control of polarisation selection with almost no insertion loss. A near-field metasurface based steering system is also presented and applied to the array for one- and twodimensional beam steering. The results are verified through model simulations and measurement of the fabricated prototypes.
\end{abstract}

Index Terms-Microstrip Antenna Array, Shared-Aperture Array, Polarisation Reconfigurable Antenna Array, Low CrossPolarisation, Linear Polarisation, Circular Polarisation.

\section{INTRODUCTION}

$\mathrm{M}$ ICROSTRIP antenna arrays have been widely used for several purposes since their inception in 1952 [1]. Ranging from RADAR to cellular and satellite communication microstrip antenna arrays have been extensively researched. Its several design aspects have also been investigated in the $20^{\text {th }}$ century and continue to be under investigation. One such design aspect is the polarisation of the array. Due to polarisation mismatch a considerable amount of signal loss occur in the receiving antennas. The ability of switching between linear and circular polarisations and also their senses can be utilized for the reduction of polarisation mismatch losses in portable devices [2] [3]. With the explosion of wireless applications, the Electromagnetic (EM) bandwidth is becoming scarce day by day. One of the many methods to overcome the EM bandwidth

This paragraph of the first footnote will contain the date on which you submitted your paper for review. It will also contain support information, including sponsor and financial support acknowledgment. For example, "This work was supported in part by the U.S. Department of Commerce under Grant BS123456." limitation is the utilisation of an antenna or array's polarisation. Different polarisations of the same antenna can be used as separate communication channels [4] to increase the effective communication capacity. This enhances the communication capacity of the antenna while using limited bandwidth, using the same resonant frequency which also adds to the diversity of antenna utilisation in multiple applications. Moreover, it has been reported in literature, e.g., [2], that interference and multipath fading can also be minimized by using flexible polarisations. Certain applications may require the reconfiguration of the antenna's polarisation online or offline to meets its specific requirement. Therefore, the reconfigurable antennas have also been actively investigated by the research community.

The two most widely used polarisations are linear polarisation (horizontal, vertical, or slant) and circular (right- or left-handed) polarisation. In literature, several approaches have been used to achieve polarisation re-configurability. Some of these methods include the use of active switching devices such as varactors or pin diodes to switch among different components of an antenna or to activate a particular section of an antenna array that corresponds to a certain polarisation [2] [5] [6] [7]. One disadvantage of active component-based switching is the insertion loss that is introduced by the switches within the antenna aperture. Another way to achieve polarisation reconfigurability is to use specially designed metasurfaces that alter polarisation of the radiation passing through them. Such surfaces are placed on top of the antenna and then rotated or moved in a certain way to achieve the polarisation reconfigurability. Few such examples are presented in [8] [9] [10]. Obviously, metasurface based polarisation reconfigurable systems need an additional item, the metasurface, to alter the polarisation. This adds to the cost and size of the antenna and may even reduce the portability of the antenna in some cases.

The next few paragraphs should contain the authors' current affiliations, including current address and e-mail. For example, F. A. Author is with the National Institute of Standards and Technology, Boulder, CO 80305 USA (email: author@ boulder.nist.gov). 
All the aforementioned benefits of polarisation reconfigurability can be achieved by using a shared aperture approach where two or more subarrays are integrated into the same aperture thus maximizing the use of available space. The shared-aperture approach eliminates the need of switching devices or metasurfaces and thus eliminates the issues associated with those techniques. Several examples exist in literature where shared aperture antennas have been reported such as [11] [12] [13] [14]. However, the focus in these papers is either on dual band operation or they use common feeding mechanism for the whole aperture. In our design, our focus is on the independent feeding on the two orthogonal subarrays to reduce coupling as well as provide independent operational capability of the subarrays. Moreover, our design can switch among various polarisation without the use of onboard switches by simple excitation control from outside the aperture.

We will explain the design of our shared-aperture array in more details in section II followed by the results and discussion in section III. Then a beam steering solution is provided in section IV which uses a pair of near-field steering surfaces (NFSS). We conclude our paper in section V.

\section{SHARED-APERTURE ARRAy CONFIGURATION}

The array consists of two subarrays that are aligned along the $x$ - and $y$-axis corresponding to horizontal and vertical linear polarisation respectively. A conceptual design is shown in Figure 1. The individual elements are microstrip square patches. The subarrays are co-axial i.e., have the same central point. The diagonal elements of the overall array are removed to maintain symmetry between the two subarrays. Moreover, to avoid a null at the centre, the central four elements are shared between the two subarrays by dual-feeding them for both polarisations.

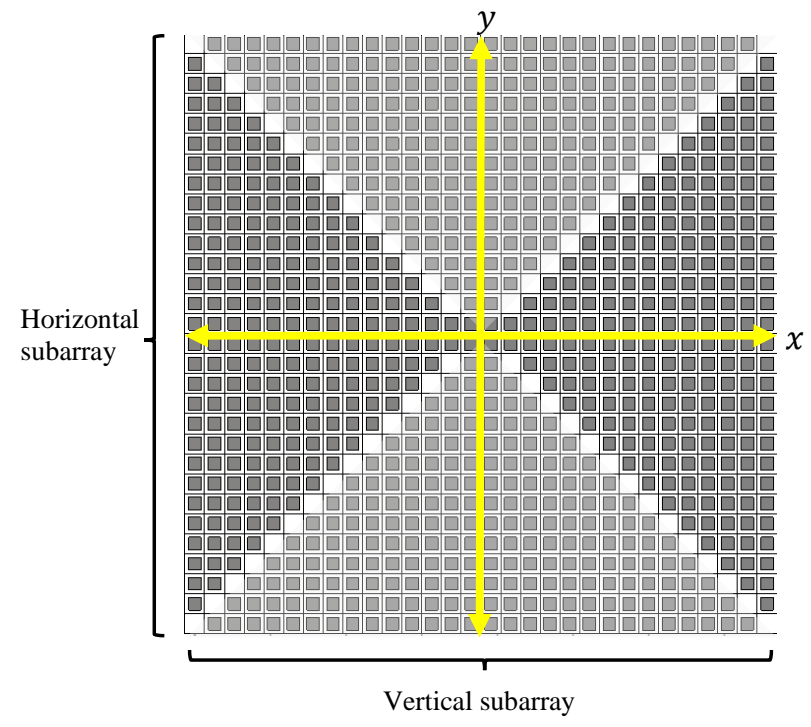

Figure 1. Shared aperture concept for polarisation reconfigurability. The light and dark grey colours represent horizontal and vertical subarrays respectively.

In order to achieve polarisation diversity, the array should be able to radiate with both linear (LP) and circular polarisations
(CP). Let us revisit the mechanism of producing various polarisations for a given electromagnetic (EM) radiation. In order for the polarisation of EM wave to be linear, only one component of the electric field (E-field) of the EM wave should be active while the orthogonal component should be zero [15]. For example, for linear vertical polarisation the E-field's xcomponent of the EM wave should be zero and, similarly, for horizontal polarisation the E-field's y-component should be zero. For linear polarisation at $45^{\circ}$ both the E-field's components should have equal amplitude with no phase difference. Likewise, to produce a circular polarisation, both the E-field's components of the EM wave should have equal amplitude but with a phase difference of $+90^{\circ}$ or $-90^{\circ}$ for righthand or left-hand circular polarisations respectively. This concept is shown in Figure 2 (a) - (f).

The elements in the two subarrays are fed such that the horizontal subarray radiates with horizontal linear polarisation while the vertical subarray radiates with vertical linear polarisation. The patch elements in the horizontal subarray are fed on the left vertical edge that causes the patch to radiate its E-field along the x-axis. Likewise, the patch elements in the vertical subarray are fed along the bottom horizontal edge because of which the patches radiate their E-field along the yaxis resulting into vertical linear polarisation.

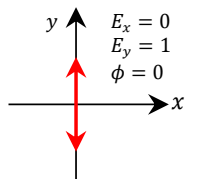

(a)

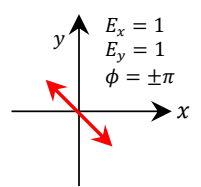

(d)

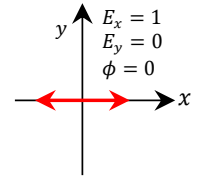

(b)

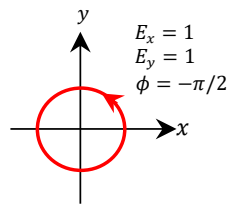

(e)

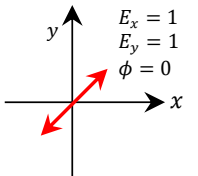

(c)

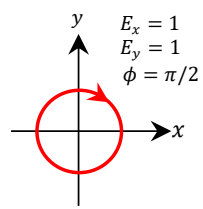

(f)
Figure 2. Electromagnetic wave polarisation as seen when the wave is approaching the viewer: (a) linear vertical polarisation, (b) linear horizontal polarisation, (c) linear polarisation at $45^{\circ}$ angle, (d) linear polarisation at $135^{\circ}$ angle, (e) right-hand circular polarisation, and $(f)$ left-hand circular polarisation [15].

To produce LP at $45^{\circ}$ we simply power both the subarray simultaneously with equal amplitude without any phase delay between them, thus, satisfying the condition in Figure 2 (c). However, for $135^{\circ}$ linear polarisation there should be a phase difference of $180^{\circ}$ between the two subarrays while maintaining amplitude the same. For circular polarisation we power both subarrays simultaneously with equal amplitude but with a phase difference of $+90^{\circ}$ for right-hand circular polarisation and of $90^{\circ}$ for left-hand circular polarisation. In practice this covers all the polarisation being used by communication devices. Now to be more specific we will discuss a $6 \times 6$ microstrip array that has been designed and fabricated with this approach followed by results and discussion.

\section{A. A $6 x 6$ Array Realisation}

In order to implement the concept discussed in previous 
section we designed a 6x6 microstrip array in CST Microwave Studio as shown in Figure 3. The array has two substrate layers; the top layer is used for the radiating elements and the lower one is used for accommodating the feed network which would otherwise be very difficult to accommodate on the same layer along with the radiating patches. The ground is sandwiched between the two substrate layers as shown in Figure 3 (e). Each subarray is to be powered separately through a microstrip feed network that is, in turn, powered by a single coaxial cable.

The elements of the array are square patches of side length $8.33 \mathrm{~mm}$ and were designed and optimized to resonate at 11.5 $\mathrm{GHz}$ frequency. Although the original goal was to have totally independent feed networks for both the subarrays, however, to avoid a null at the centre of the array the central four elements need to contribute to both polarisations. Therefore, they were fed by both the horizontal and vertical feed networks. The detail of the feed network design will be given next.

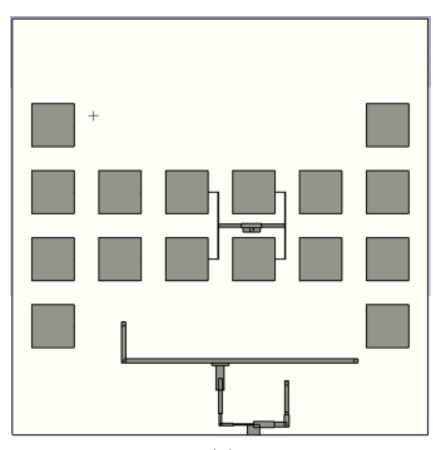

(a)

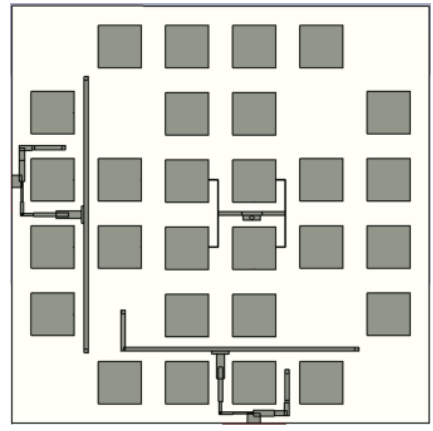

(c)

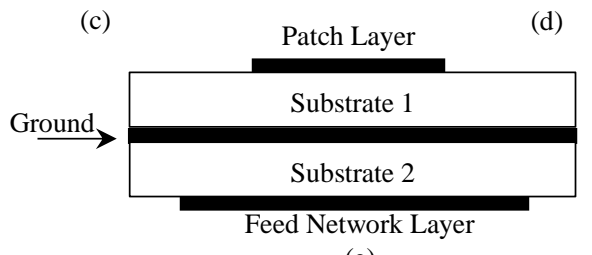

(e)

Figure 3. A $6 x 6$ shared aperture array: (a) the horizontal subarray, (b) vertical subarray, (c) full array with horizontal and vertical subarrays combined, (d) the array feed network on the lowest layer, and $(e)$ the side view of the array showing different layers.

\section{B. Feed Network Design}

The feeding mechanism for the array is a bit tricky as the geometry of the array is irregular, i.e., it is neither strictly linear nor rectangular. To explain the feeding design, we will consider vertical subarray (shown in Figure 3 (b)) as an example. The horizontal subarray basically uses similar feed design but rotated by $90^{\circ}$. The total number of elements in the subarray is 16. If we choose the traditional equal power distribution for feed network it will result into high sidelobe level and wider beamwidth due to irregular shape of the array. Since the array's geometry bears a resemblance more to a linear array, we choose Taylor's tapering techniques that is well-known for low sidelobe levels [16].

The power coefficients for individual elements of the array are calculated according to the Taylor's window given below:

$$
w(t)=\left[1+2 \sum_{m-1}^{\bar{n}-1} F(n, A, \bar{n}) \cos 2 \pi m t\right] \operatorname{rect}(t)
$$

Various components used in the above equation are defined as follows:

$$
\begin{aligned}
& F_{m}=\frac{-\left(\frac{(-1)^{m}}{2}\right) \prod_{n=1}^{\bar{n}-1}\left[1-\frac{\frac{m^{2}}{\sigma^{2}}}{A^{2}+\left(n-\frac{1}{2}\right)^{2}}\right]}{\prod_{n=1, n \neq m}^{\bar{n}-1}\left[1-\frac{m^{2}}{\sigma^{2}}\right]} \\
& \sigma^{2}=\frac{(\bar{n})^{2}}{A^{2}+\left(n-\frac{1}{2}\right)^{2}} \\
& A=\frac{a \cosh \eta}{\pi}=\frac{\ln \left(\eta+\sqrt{\eta^{2}-1}\right)}{\pi} \\
& \eta=10^{-\frac{S L L}{20}}
\end{aligned}
$$

where SLL is the desired side lobe level relative to the main lobe in $\mathrm{dB}$ maintained over a distance $\bar{n}$ from the main lobe.

Using the Taylor's window equations (1) - (5), we calculated the power coefficients for all the elements in the vertical subarray which are shown in Figure 4.

In Figure 4, we can see that subarray has higher power division ratio for exterior lines for which designing a power divider will be a challenge as high division ratio will cause one arm of the power divider to be very narrow to an extent that it could not be fabricated due to limited resolution of the available fabrication technology. Moreover, 3-way power dividers will be needed to distribute power from one line to another which will further complicate the power division and increase mismatch losses. To avoid such a situation, we rearranged the power division such that the whole feed network will use only 2-way power dividers. Additionally, the new arrangement makes sure that the majority of the network utilise equal power division with ratio of 1 reducing the number of non-equal power dividers to only three. Equal power dividers are also referred to as splitters. This new arrangement of feed network is shown in Figure 5. 


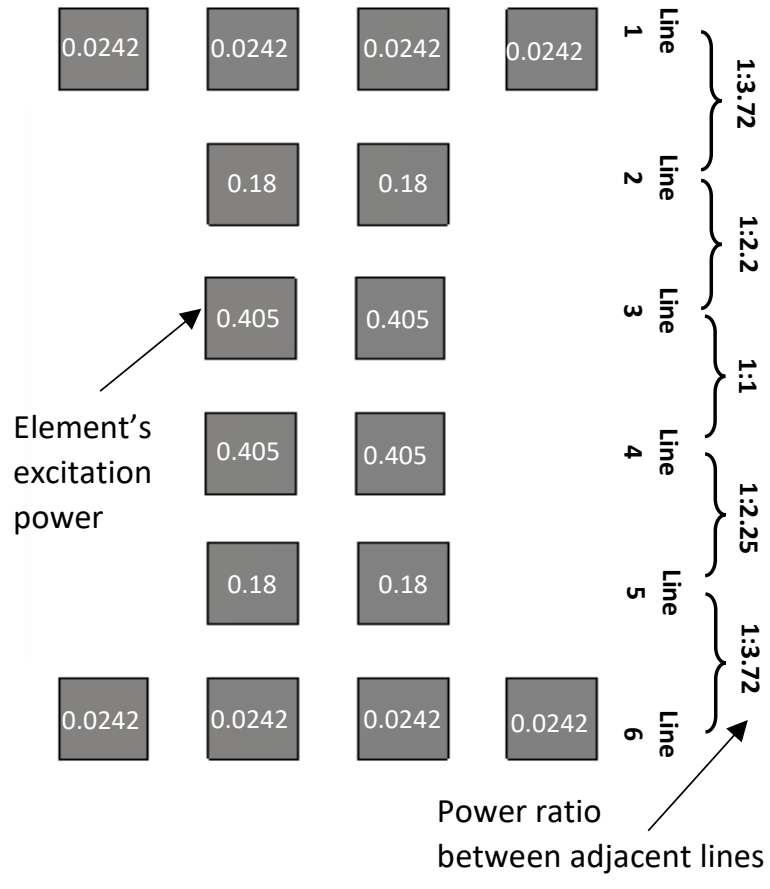

Figure 4. The power coefficients for individual elements of one subarray according to Taylor's tapper.

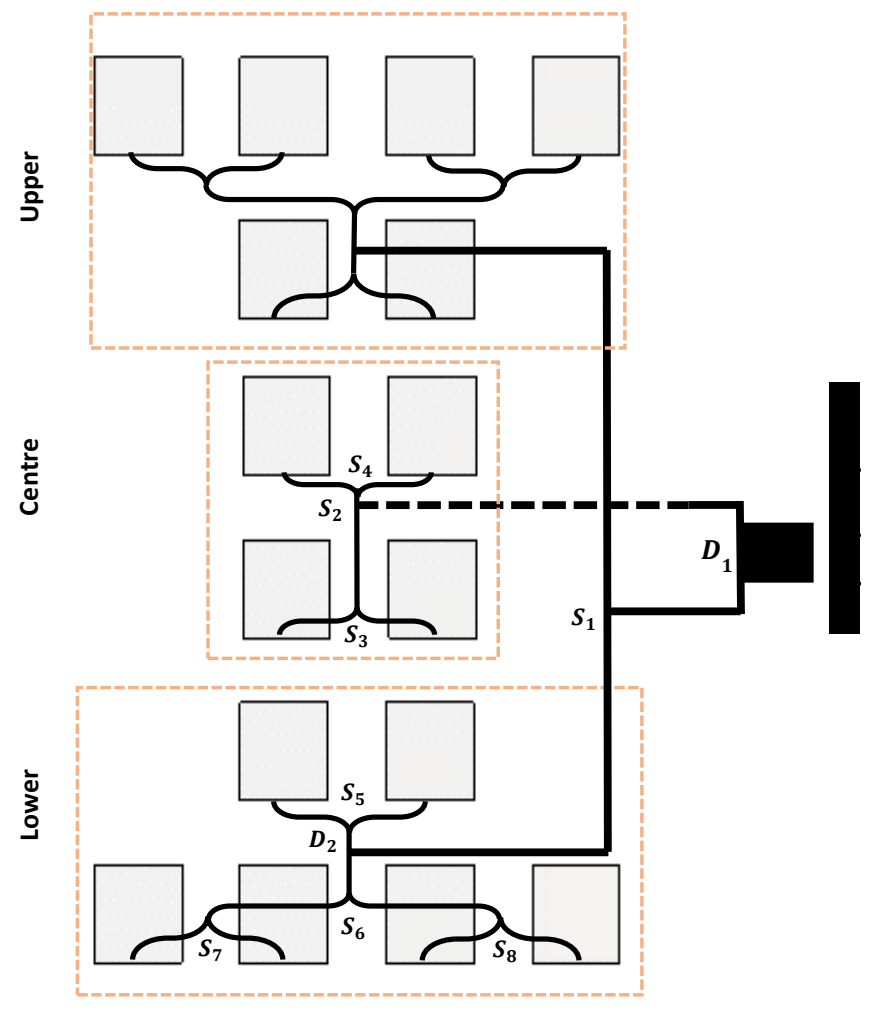

Figure 5. Rearranged feed network having no 3-way dividers. $\boldsymbol{S}$ stands for splitter while $\boldsymbol{D}$ for divider. Majority of the network consists of splitters avoiding high power unequal division ratios.

\section{RESULTS AND DISCUSSION}

In this section we are going to explain with brevity how each polarisation can be produced along with the simulation and measured results for each polarisation.

\section{A. Linear Polarisation}

For linear polarisation (LP) only one subarray needs to be activated. For example, for linear vertical polarisation, only the vertical subarray is switched on while the horizontal subarray is kept inactive. Likewise, only horizontal subarray is powered on for horizontal linear polarisation while keeping the vertical subarray inactive. To achieve $45^{\circ}$ and $135^{\circ} \mathrm{LP}$, both subarrays must be excited simultaneously. After applying these settings, we simulated the array in CST Microwave Studio for each case. Figure 6 shows the electric field distribution of the array when it was simulated for various linear polarisations. The E-field distributions for the horizontal and vertical polarisation are given in Figure 6 (a) and (b) while the same for $\mathrm{LP} 45^{\circ}$ and $135^{\circ}$ are given in Figure 6 (c) and (d) respectively. It is evident from Figure 6 (a) - (d) that the polarisations were correctly produced by the array with the aforementioned settings.

Now we will present the radiation patterns and other relevant results for the linear polarisation cases. The scattering parameter S11 plots for individual subarrays are given in Figure 7 where we see both subarrays resonate very well at the intended frequency of $11.5 \mathrm{GHz}$ with reflection less than -23 $\mathrm{dB}$ and $-26.8 \mathrm{~dB}$ for horizontal and vertical subarrays respectively.

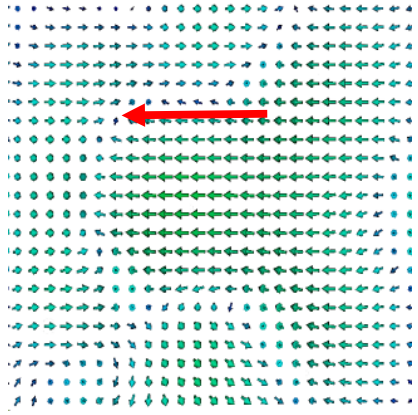

(a)

खx

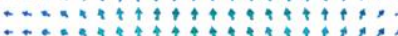
$\therefore \ldots$ O

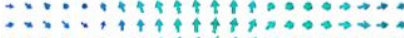

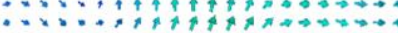

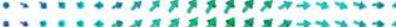

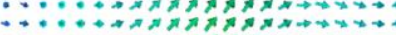

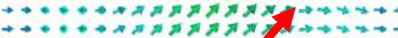

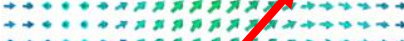
$\vec{A}: \therefore$ :

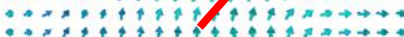

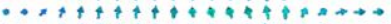

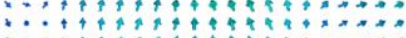
(c)

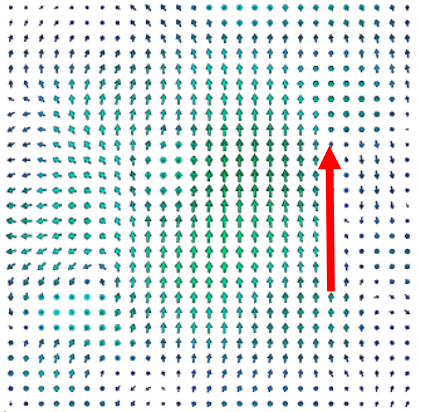

(b)

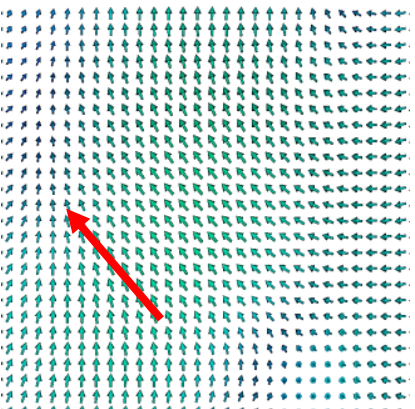

(d)
Figure 6. Electric field distribution of the array at $11.5 \mathrm{GHz}$ for various cases: (a) linear horizontal subarray only, (b) linear vertical subarray only, (c) when both subarrays were excited simultaneously for $45^{\circ} \mathrm{LP}$ and (d) for $135^{\circ} \mathrm{LP}$ respectively. 


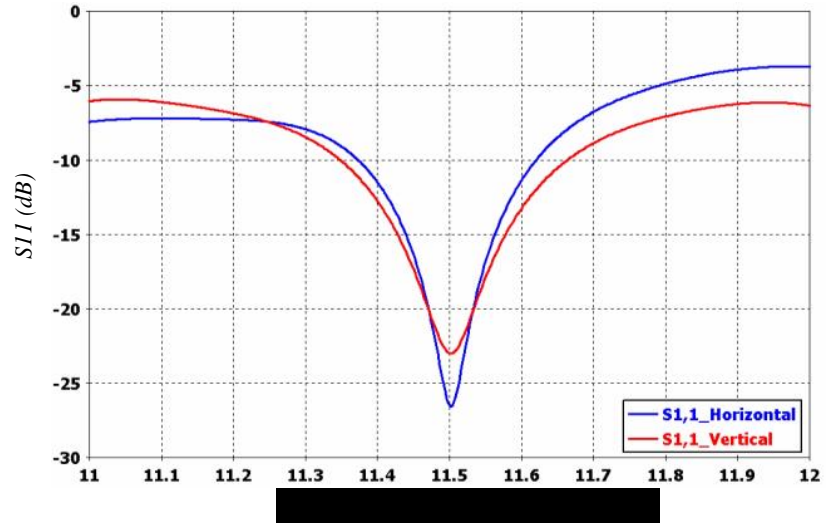

Figure 7. S11 plot for individual excitation of the horizontal (solid curve) and vertical (dashed curve) subarrays.

The far-field radiation patterns (directivity) of the horizontal array obtained from simulation in CST Microwave Studio are given in Figure 8 for azimuth angle $\phi=0^{\circ}$ and elevation angle $\theta=0^{\circ}$ corresponding to broadside normal direction which was the original intended direction of the designed array. The patterns were computed for the bandwidth of interest (11- 12 $\mathrm{GHz}$ ). The radiation maintains very similar patterns in the 11 $12 \mathrm{GHz}$ band with very minor variation which means the array operation is very stable in this frequency range. Additionally, the array beamwidth is also sufficiently narrow given the irregular shape of the array. Moreover, the E-field distribution shown in Figure 6 (a) demonstrated that the emitted radiation has linear horizontal polarisation.

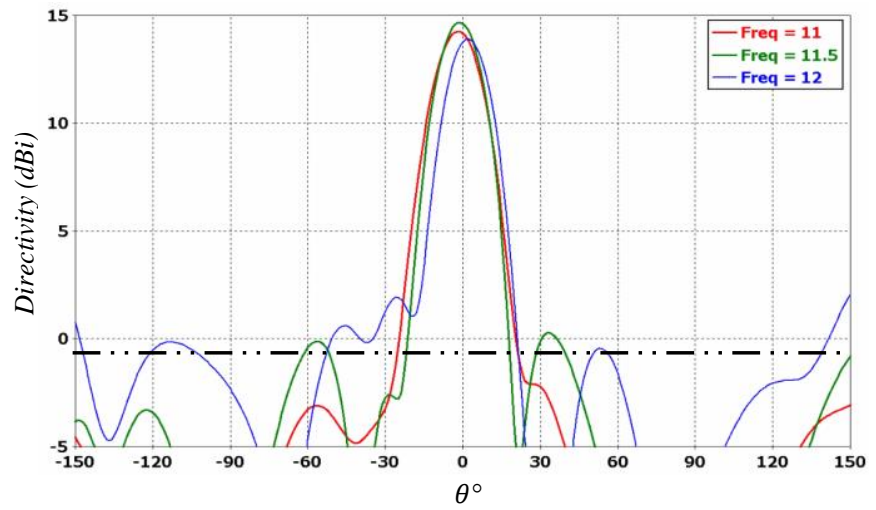

Figure 8. Far-field radiation pattern (directivity) of the horizontal subarray in broadside $\left(\phi=0^{\circ}, \theta=0^{\circ}\right)$.

Similarly, we computed the broadside radiation directivity patterns for the vertical subarray by simulating the array in the CST Microwave Studio and these patterns are given in Figure 10 for the $11-12 \mathrm{GHz}$ bandwidth. Also, the E-field distribution shown in Figure 6 (b) shows that the vertical subarray correctly produced vertical linear polarisation. The directivity bandwidth of both subarrays was also evaluated. Figure 9 shows the peak directivity of both subarrays in the broadside direction across the frequency band $10.5-12.5 \mathrm{GHz}$ where we see that both subarrays maintain a good directivity level with less than $-3 \mathrm{~dB}$ drop in the specified band.

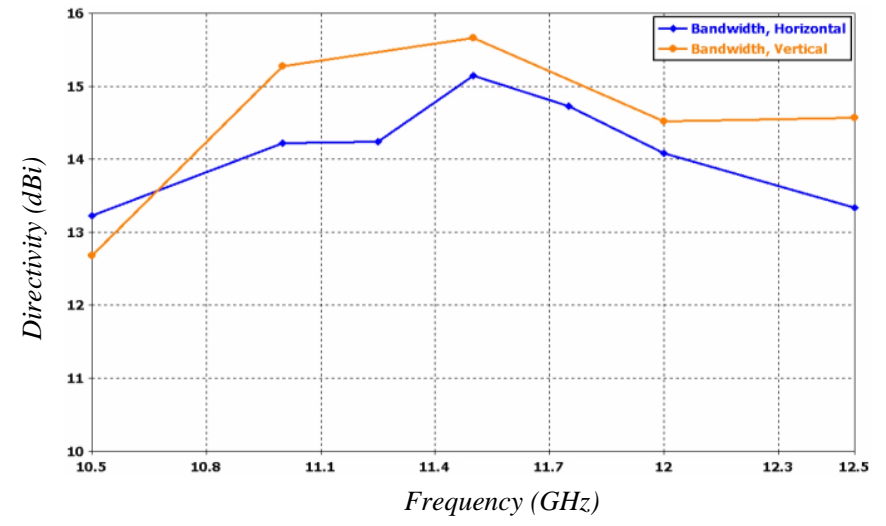

Figure 9. Array's peak directivity in the broadside direction within 10.5 - $12.5 \mathrm{GHz}$ band.

In order to see the polarisation purity, we have plotted the copol and cross-pol components in Figure 11 and Figure 12 for the horizontal and vertical subarrays respectively. From these figures we see that both the subarrays were able to maintain at least $20 \mathrm{~dB}$ difference between the co-pol and cross-pol components in all the given cases. The horizontal subarray has about $43 \mathrm{~dB}$ isolation between co-pol and cross-pol components in the direction of the main beam.

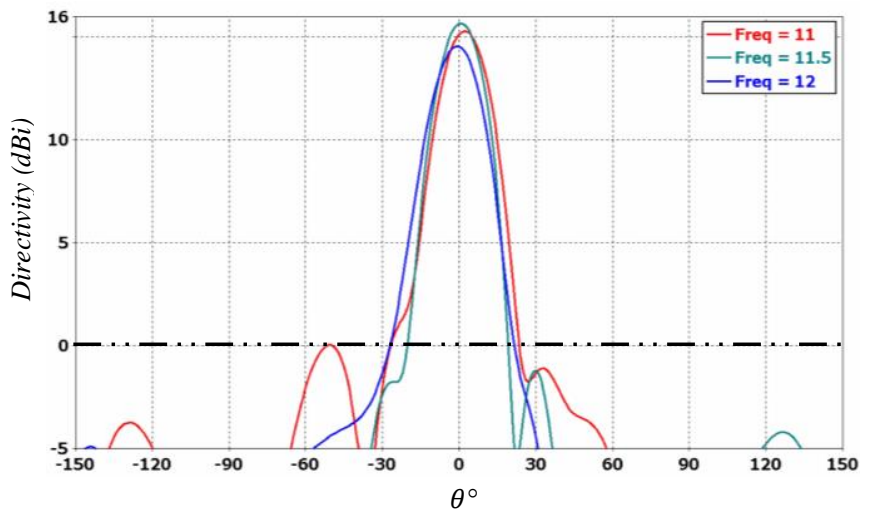

Figure 10. Far-field radiation pattern (directivity) of the vertical subarray in broadside $\left(\phi=0^{\circ}, \theta=0^{\circ}\right)$.

We, then, simulated the array for the $45^{\circ}$ and $135^{\circ}$ linear polarisations. This is achieved by exciting both the horizontal and vertical arrays simultaneously while keeping a phase difference of $0^{\circ}$ and $+180^{\circ}$ to the input of horizontal subarray for the linear $45^{\circ}$ and $135^{\circ}$ polarisations respectively. The farfield radiation directivity patterns for the $45^{\circ} \mathrm{LP}$ are given in Figure 13. In the case of $45^{\circ} \mathrm{LP}$, the array was able to maintain a directivity between 14.8 and $15 \mathrm{~dB}$ for all azimuth angles at broadside within the frequency band $11-12 \mathrm{GHz}$. The sidelobe level (SLL) is restricted to $-16 \mathrm{~dB}$ for all azimuth angles. Patterns for frequencies with in this ranges are very similar which demonstrates that the array's operation is very stable within $11-12 \mathrm{GHz}$, a desirable characteristic. 


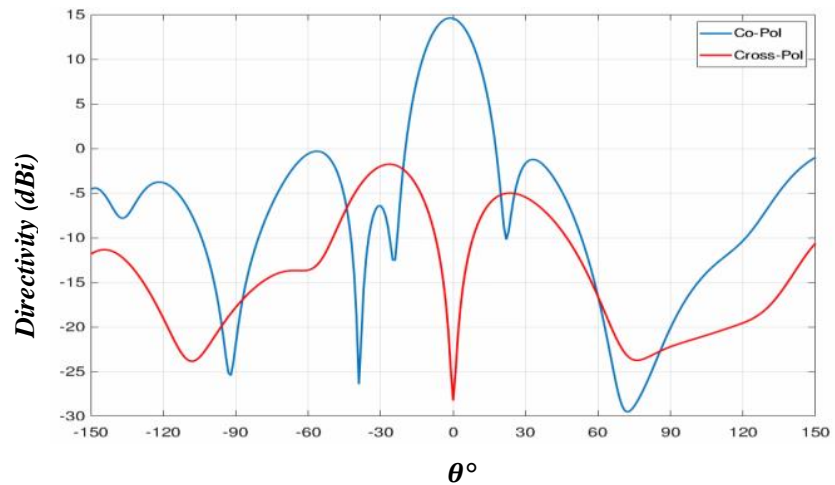

Figure 11. Co-pol and cross-pol patterns of the horizontal subarray at $11.5 \mathrm{GHz}$.

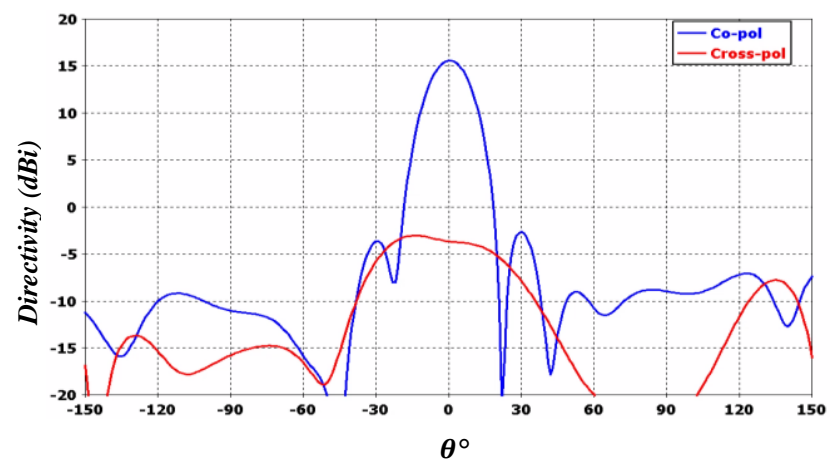

Figure 12. Co-pol and cross-pol patterns of the vertical subarray at $11.5 \mathrm{GHz}$.

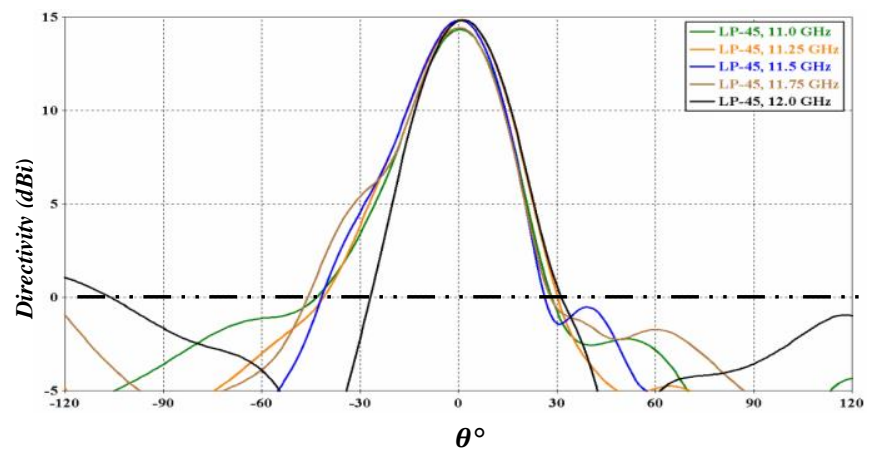

(a)

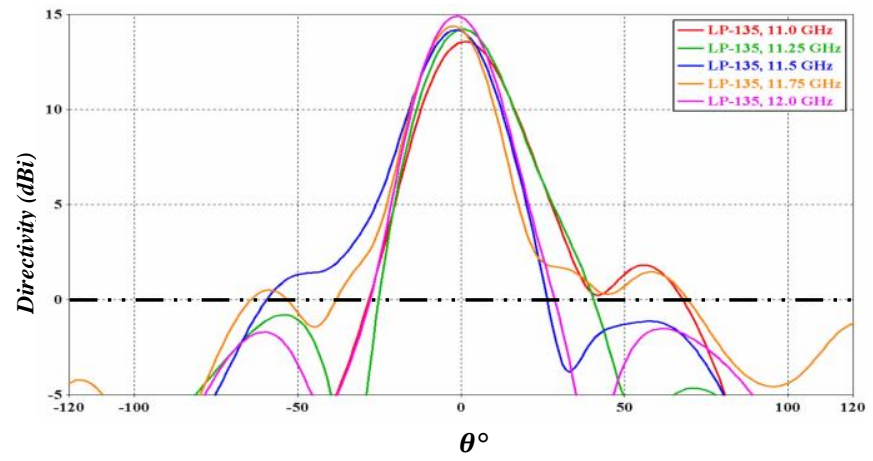

(b)

Figure 13. Far-field directivity pattern of the array for (a) $45^{\circ} \mathrm{LP}$ and (b) $135^{\circ} \mathrm{LP}$ for various frequencies within $11-12 \mathrm{GHz}$.

The far-field radiation directivity patterns for $135^{\circ} \mathrm{LP}$ are given in Figure 13 (b) where it is evident that the array can achieve directivity between 14.7 and $15 \mathrm{~dB}$ with a maximum SLL of value $-13.4 \mathrm{~dB}$ for all azimuth angles in the broadside direction within the frequency band $11-12 \mathrm{GHz}$. It is worth mentioning that the array not only maintains the directivity level but also the beam shape within $11-12 \mathrm{GHz}$.

During dual-port operation the port isolation (S21 and S12) observed during simulation is given in Figure 15 where we see that for the most part of the frequency band of interest the port isolation is better than $-26 \mathrm{~dB}$ and goes as low as $-30 \mathrm{~dB}$. At very few specific frequencies it is $-24 \mathrm{~dB}$.

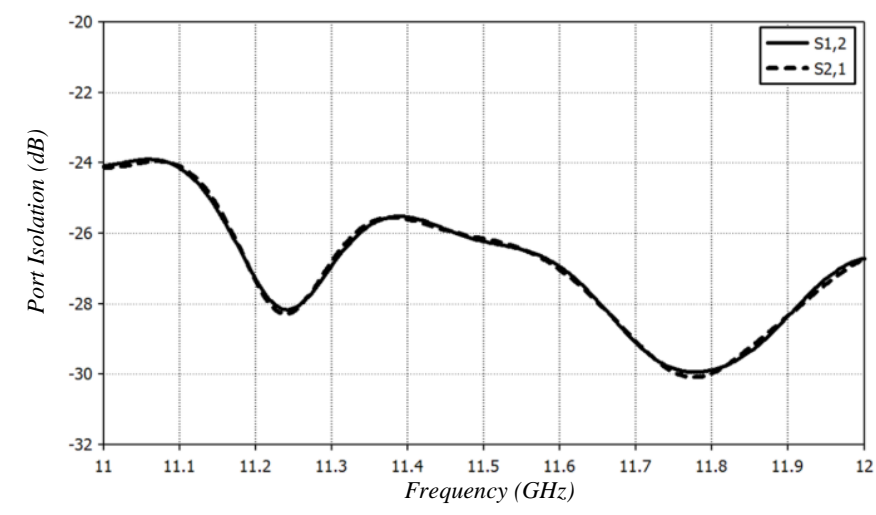

Figure 15. Port isolation when each subarray is used individually.

\section{B. Circular Polarisation}

For circular polarisation, we have to fulfill the requirement in Figure 2 (e) and (f) for left-hand and right-hand circular polarisations. Therefore, we simulated both subarrays by exciting simultaneously, with appropriate phase difference between their excitation, but with equal amplitudes. The resulting far-field directivity radiation patterns are given in Figure 17 (a) for LHCP and in Figure 17 (b) for RHCP. In both cases the array maintains very similar radiation patterns with maximum directivity around $15 \mathrm{~dB}$ for various frequencies within $11-12 \mathrm{GHz}$ band. Moreover, the radiation pattern does not deteriorate within this narrow band and has no significant sidelobe level.

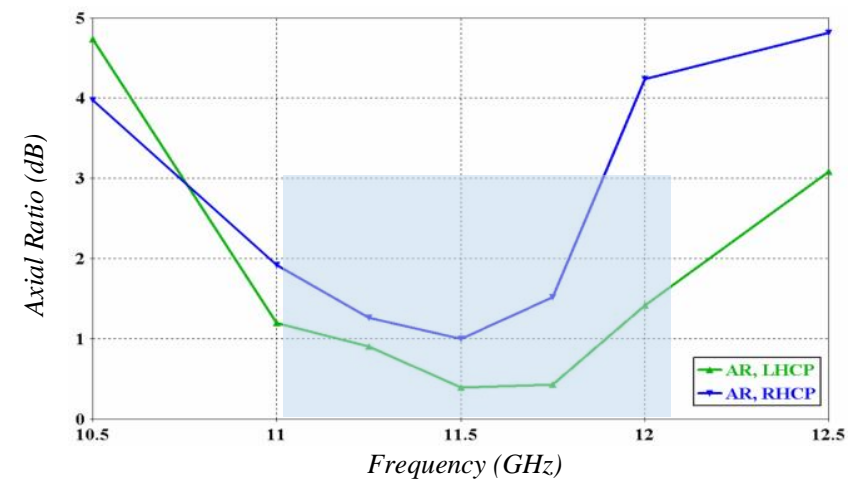

Figure 16. Axial ratio vs frequency plot of the array for $L H C P$. 


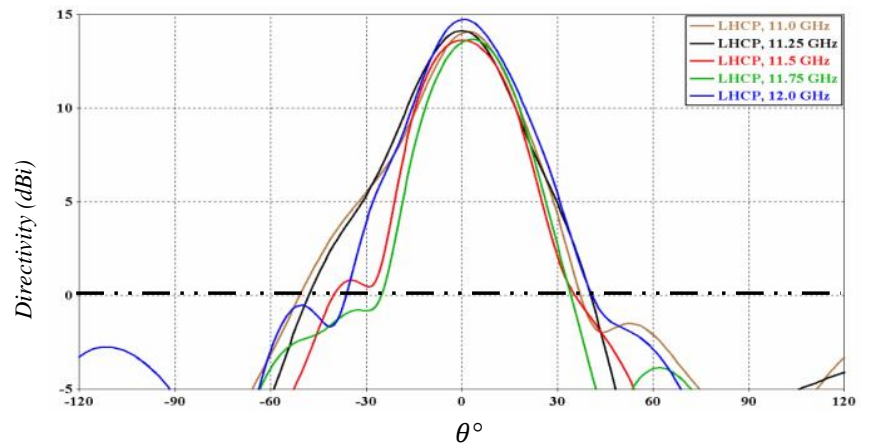

(a)

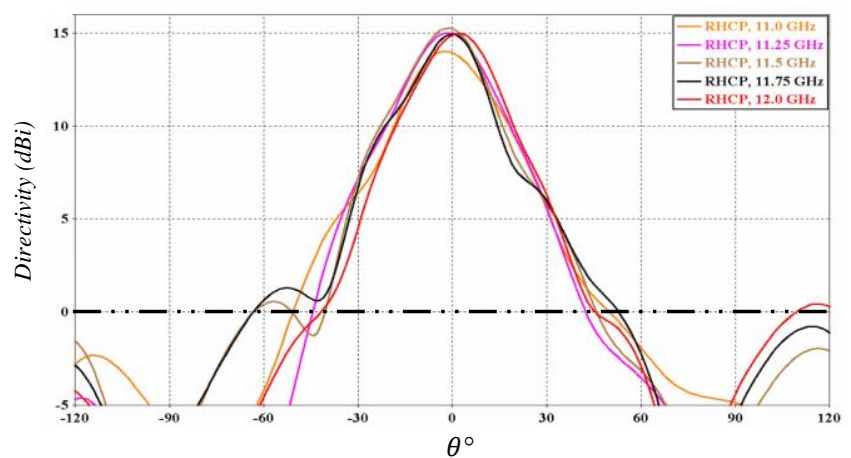

(b)

Figure 17. Far-field directivity pattern of the array for (a) LHCP and (b) RHCP frequency range of $11-12 \mathrm{GHz}$.

We computed and plotted the axial ratio of the array in Figure 16 when it was operated in LHCP and RHCP modes. The axial ratio $(\mathrm{AR})$ is $0.5 \mathrm{~dB}$ and $1 \mathrm{~dB}$ at the resonant frequency of 11.5 $\mathrm{GHz}$ for LHCP and RHCP polarisation respectively. Moreover, the AR remains below $3 \mathrm{~dB}$ for the most part of the $11-12$ $\mathrm{GHz}$ band indicating good circular polarisation except for very few frequencies at the higher end of the band.

\section{Fabrication and Measurement}

The array was fabricated in a commercial facility with a 0.1 $\mathrm{mm}$ accuracy and is shown in Figure 18 where the top side of array is presented in part (a) whilst part (b) shows the bottom side of the array containing the feed network. For inputs, $50 \Omega$ SMA connectors were used. The fabricated prototype was then measured in an anechoic near-field measurement chamber using NSI2000 setup.

The subarrays were excited one by one and measured for LP whilst for $\mathrm{CP}$ measurement a common input was used with a $90^{\circ}$ phase shifter splitting the signal for subarrays for LHCP. The inputs to the array coming from the phase shifter were switched (producing $-90^{\circ}$ phase difference) to generate RHCP. Figure 19 shows the measurement setup for both LP and CP measurement in an anechoic chamber. All the subsequent measurement results will be presented for the broadside direction, i.e., $\theta=\phi=0^{\circ}$. Moreover, two orthogonal plane cuts will be given for each case. These cuts are $\mathrm{H}$-plane and Vplane. $\mathrm{H}$-plane cut is along the $\mathrm{x}$-axis while $\mathrm{V}$-plane cut is along $\mathrm{y}$-axis. The array was placed vertically as shown in Figure 19 such that its broadside faces the radiation coming from the probe. The waveguide probe used for measurement is WR-75, model ANT-WGP-10-15 from NSI-MI Technologies having an operating frequency range of $10-15 \mathrm{GHz}$. The probe was operated in both polarisations LP-0 and LP-90 during the nearfield measurement.

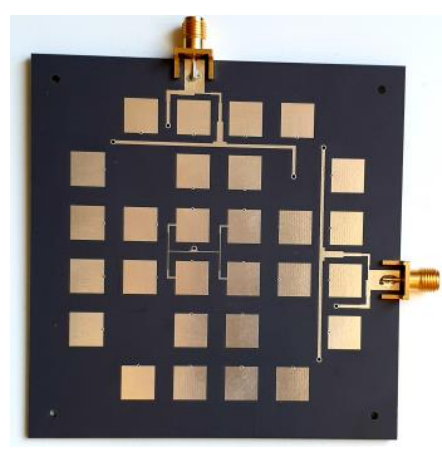

(a)

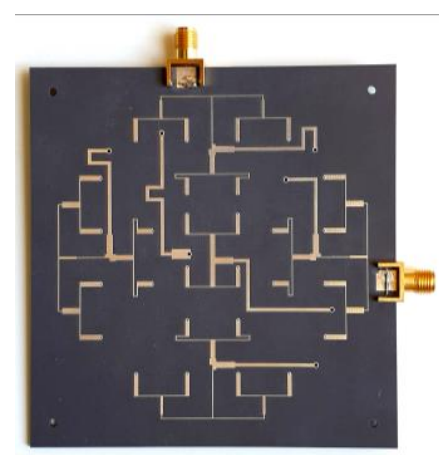

(b)
Figure 18. Fabricated prototype of the array: (a) top view and (b) bottom view.

The measured radiation patterns of the array at $11.5 \mathrm{GHz}$, when only horizontal subarray was excited, are shown in Figure 20. The patterns agree well with the simulation results presented in Figure 8. The patterns are wider in the V-plane because most of the subarray elements are aligned along the $\mathrm{H}$ plane. The sidelobe level is very similar to the simulation results.

The array was then operated for vertical LP by exciting the vertical subarray only and its radiation patterns are given in Figure 21 where we see that the patterns along the subarray's plane (V-plane in this case) are quite similar to the simulation results presented in Figure 10 with slightly higher SLL.

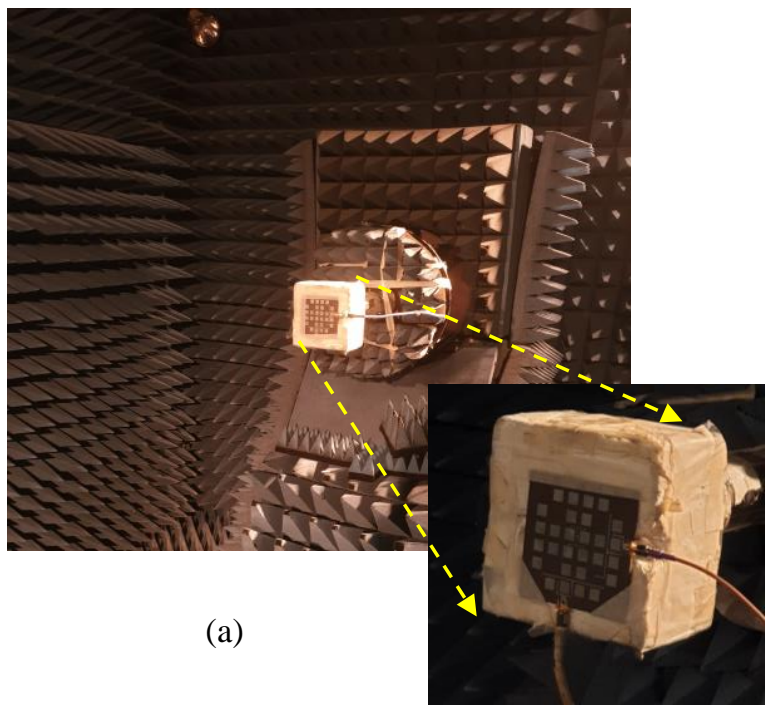

(b)

Figure 19. Array's measurement setup for: (a) LP excitation and (b) $C P$ excitation. 


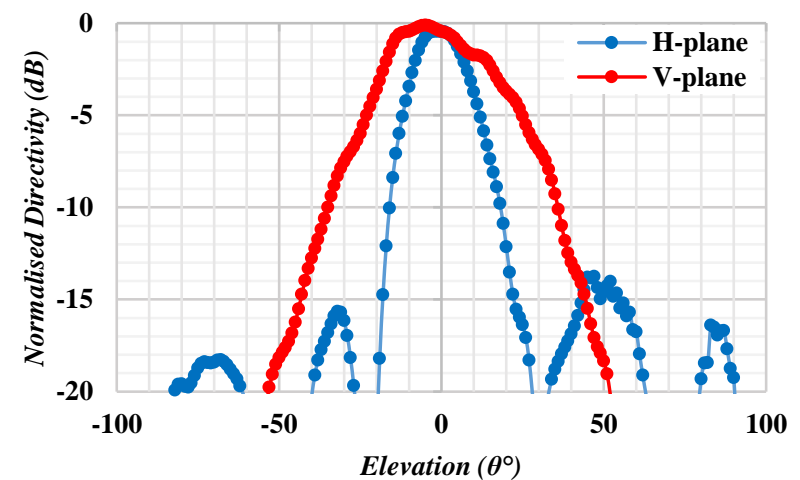

Figure 20. Measured directivity patterns of the horizontal subarray.

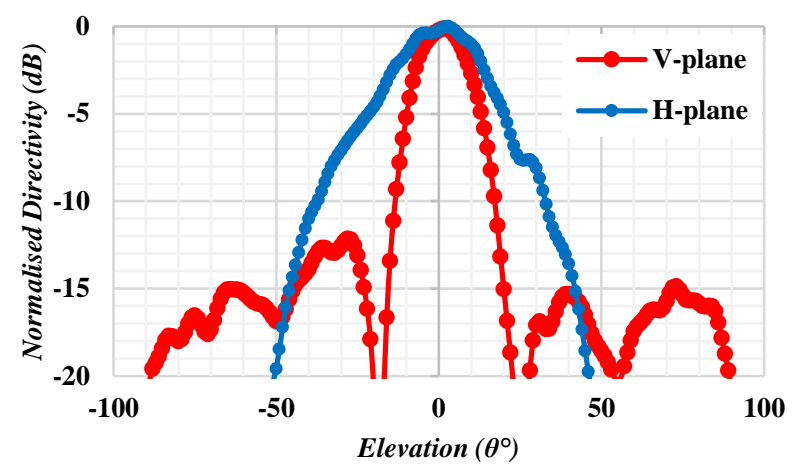

Figure 21. Measured directivity patterns of the vertical subarray.

The measured radiation pattern (directivity) for LHCP and RHCP are given in Figure 22 where we see the array's radiation is symmetrical in both $\mathrm{H}$ and $\mathrm{V}$ planes. This is because now both subarrays are radiation simultaneously with equal number of elements along both axes creating a pencil beam shape. The array is able to maintain a narrow beam with good sidelobe level. For RHCP, we switched the inputs to the array coming from a splitter with $90^{\circ}$ phase difference between the two splits. This reverses the phase difference in the inputs and hence a reversal in CP sense occurs. The measured results for RHCP are in shown in Figure 23. Here, in this case, the SLL is slightly higher than expected in the H-plane. However, the array managed to maintain good pattern in the V-plane.

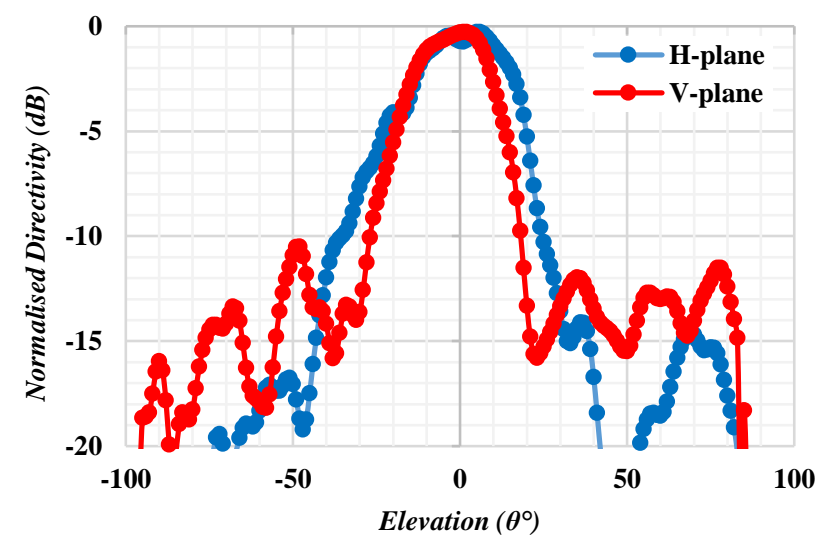

Figure 22. Measured directivity pattern cuts when array was operated in LHCP mode.

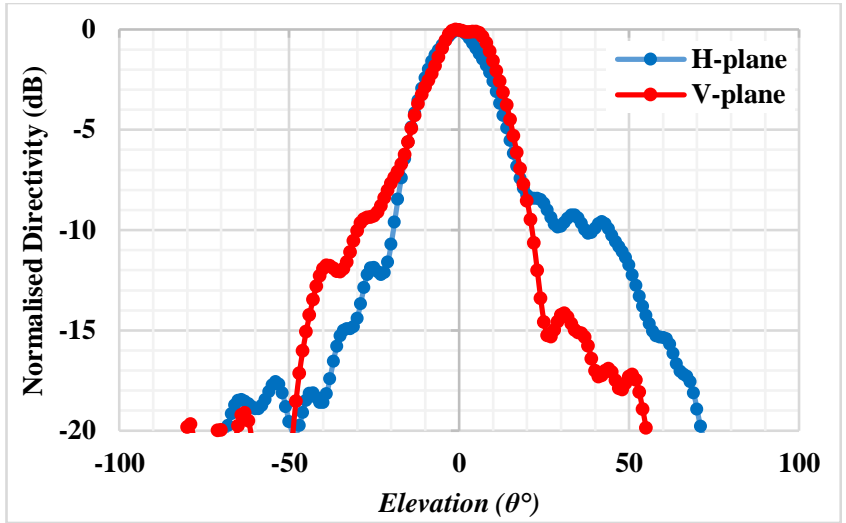

Figure 23. Measured directivity patterns for RHCP mode.

\section{Scalability}

For satellite communications high gain is required. To achieve such a high gain an array needs to have a large number of elements usually in hundreds or even thousands. Therefore, we investigated the scalability of our array design for high gain. Figure 24 shows a $32 \times 32$ version of our array's design. The size of this array is $16 \lambda_{0}$ which is $41.6 \mathrm{~cm} \times 41.6 \mathrm{~cm}$. The simulated far-field gain pattern of this design is given in Figure 25 for the resonant frequency of $11.5 \mathrm{GHz}$ where we can see a gain of 30 $\mathrm{dB}$ can easily be achieved. Moreover, the array maintains a good peak directivity in the broadside over the bandwidth of interest i.e., $11-12 \mathrm{GHz}$. The elements of the array were fed on the edges and Taylor tapering were applied to the input. The level of gain can further be improved by tuning the feed tapering. This version of the array will also be used in section IV.C for two-dimensional beam steering.

\section{Beam SteERING Using Near-Field Metasurfaces}

The designed array presented in previous section was intended to be used in satellite-on-the-move applications. As the name suggests, the array would be installed on a mobile object such as a car, train, aeroplan etc. Whenever the object changes its position so will the direction of the array's beam and might lose connectivity with satellite. Therefore, the array should have a beam steering capability to steer its beam for alignment with the satellite direction.

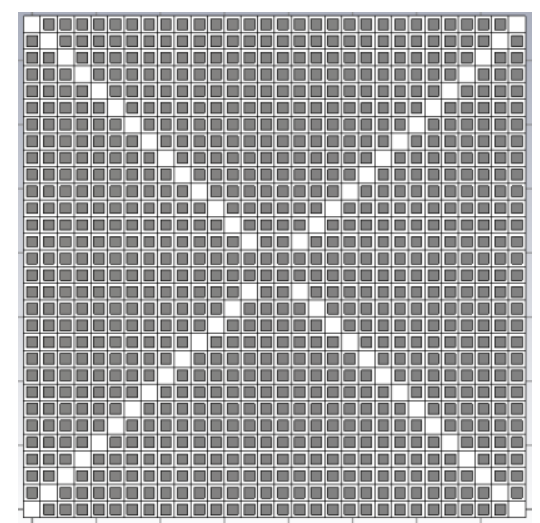

Figure 24. A $32 \times 32$ version of the array design. 


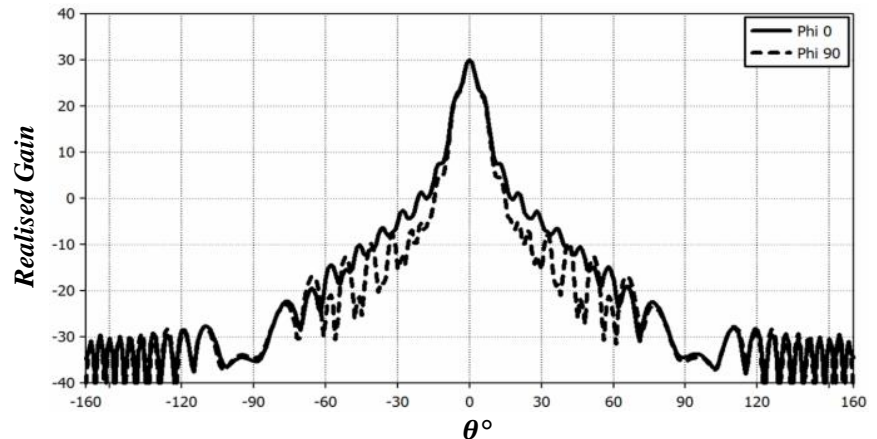

(a)

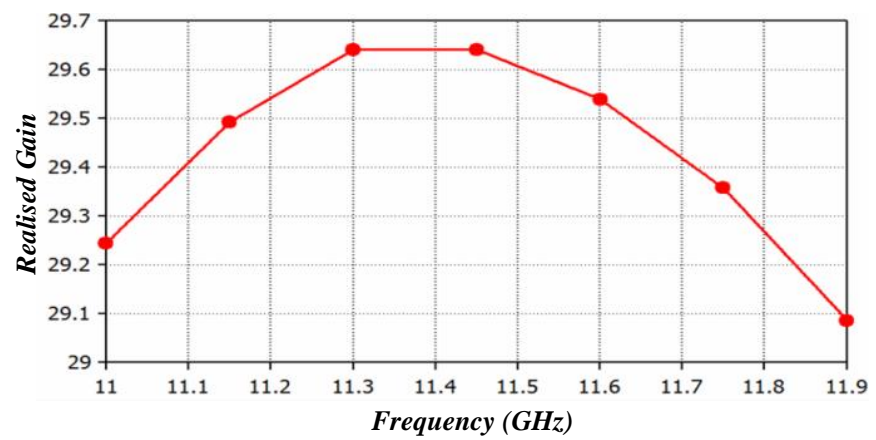

(b)

Figure 25. (a) Far-field directivity pattern of a $32 \times 32$ array at 11.5 $\mathrm{GHz}$ and (b) the broadband far-field directivity plot for $11-12 \mathrm{GHz}$.

Traditionally, the beam of a microstrip patch array is electronically steered with the use of phase shifters. However, phase shifters not only require power to operate but also are expensive and are not suitable for mass production. Over the past few decades, beam steering metasurface have been investigated for such use [17] [18] [19] [20]. Metasurface based beam steering usually involves two one-dimensional (1D) metasurfaces placed either in the near field or the far field of the base antenna or array. Such metasurfaces are made of passive meta-cells that do not require power to operate. Moreover, they are made of cost-effective substrates and copper layers that are easily available for fabrication. Of our interest are the near-field metasurfaces that can be placed very near to the base array, thus reducing the overall height of the complete array's system. There are numerous metasurface designs available in literature having various advantages and disadvantages [17] [18] [19] [20]. Since, in this section, our main goal is to demonstrate the beam steering capability of the array with the help of a nearfield steering metasurfaces (NFSS), we select the traditional square patch geometry for the design of near-field metasurfaces.

Figure 34 shows the basic setup that is used for beam steering. The setup consists of a source antenna or array (not shown) and two 1D near-field steering metasurfaces (NFSS). Each NFSS is a phase-gradient surface, that is, the surface produces a progressive phase shift in the incoming wave along its aperture. The idea of phase-gradient has been borrowed from classical linear array beam forming technique where two consecutive individual elements of the array are excited with a constant phase difference $\left(\phi_{e}\right)$ in their excitations. This phase difference is given by:

$$
\phi_{e}=\frac{2 \pi}{\lambda} d \sin \theta
$$

where $\lambda$ is the operating wavelength, $d$ is the distance between two consecutive elements which is assumed to be the same for all elements, and $\theta$ is the desired elevation angle towards which the beam of the array will be steered.

\section{A. Cell Design}

In order to design the phase-gradient NFSS we will first need to investigate the design procedure and properties of the constituent cells also called meta-cell. A typical cell consists of layers of dielectric and metal materials [18] or sometime just made of all metal [19] or all dielectric [20] depending on their application. We consider a cell design having two substrate layers sandwiched between three metal layers. The metal layer is a square patch whose dimension can be varied to obtain different properties of the cell. The substrate material chosen is RT5880 having a dielectric constant, $\epsilon_{r}=2.2$ for its properties in terms of large phase bandwidth and the capability of achieving greater range of phase shifts desired for NFSS design. The substrate thickness directly affects the transmission properties of the cell and should be selected carefully. The greater the thickness the greater the phase range that can be achieved with a unit cell. However, greater thickness results into bulky, heavy, and more cost which is not desirable specially in the communication-on-the-move (COTM) applications. Reducing the thickness will limit the phase shift range achievable with good transmission amplitude. Therefore, after careful consideration and to be consistent with antenna array design presented in previous section, the thickness of the dielectric material was selected to be $0.762 \mathrm{~mm}$ which is a standard thickness available from the Rogers Inc. The material RT5880 has a loss tangent $(\tan \delta)$ of 0.0009 which provides minimal transmission loss. The metal layer was selected to be copper for its properties and easy availability.

The next step is to build the cell in a simulation environment such as CST Microwave Studio. The surrounding environment of a cell affects its EM properties. These properties change when the surrounding environment changes due to complex field phenomena such as coupling, surface waves etc. To reduce behaviour predictability, a cell is studied in a homogeneous environment where it is surrounded by similar cells in all directions in a two-dimensional infinite plane. Basically, a periodic structure is constructed by populating the $2 \mathrm{D}$ plane with similar cells. This setup ensures the cell's environment is not affected by unpredicted media and the properties analysed are true representative of the cell. However, the construction and simulation of an infinite surface is impossible. This issue can be resolved by consulting periodic structure theory $[3,18$, 19] according to which the effect of an infinite periodic structure can be obtained by applying appropriate boundary conditions provided that the following condition is met:

$$
s<\frac{\lambda_{0}}{1+\left|\sin \theta_{\max }\right|}
$$


where $\lambda_{0}$ is the wavelength of operation, $\theta_{\max }$ is the largest angle that the incidence wave makes with the normal of the unit cell, and $s$ is the length of one side of the unit cell. These boundary conditions are applied by assuming perfect electric conductor (PEC) and perfect magnetic conductor (PMC) boundaries around the unit cell as shown in Figure 26.

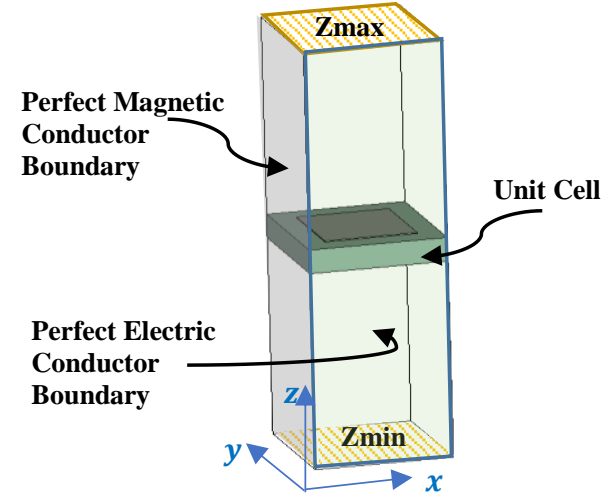

Figure 26. Boundary condition setting in CST Microwave Studio.

PEC is defined along the axis corresponding to the polarisation of the electric field of the incident wave which in our case is assumed to be along $x$-axis while PMC is defined along the magnetic polarisation direction, at right angle to PEC (y-axis). Another approach that can be taken in CST Microwave Studio is to select the option of unit cell when setting up boundaries (shown in Figure 4.4 (b)). Moreover, we assume the wave is propagating along the $z$ direction. The incident waves are generated by applying two Floquet ports in CST Microwave Studio represented as Z $\min$ and $Z \max$ as shown Figure 26. Since the size of the unit cell is very small and meets the condition in (7), the higher order Floquet modes are negligible. It was confirmed with several simulations that the appropriate number of modes for Floquet ports is 2.

In order to observe the behaviour of the cell, the metal patch dimensions are varied between minimum $(0.1 \mathrm{~mm})$ and maximum $(s-0.1=8.8 \mathrm{~mm})$ and its transmission properties are recorded for each dimension. For simplicity, the dimensions of the top and bottom layers are kept the same while the middle layers' dimension can be different from top and bottom layers. Figure 27 shows the scenario when the dimension of top and bottom patches $\left(a_{1}\right)$ was varied from $0.1 \mathrm{~mm}$ to $8 \mathrm{~mm}$ while keeping the dimension of the middle layer fixed at $a_{2}=2$. Figure 27 (a) displays the transmission phase of the unit cell with respect to $a_{1}$ while Figure 4.6 (b) shows the transmission amplitude when $a_{1}$ was varied. Figure 27 (c) - (f) displays how the unit cell looks physically when $a_{1}=0.1,2,4,6 \mathrm{~mm}$ respectively. Note that the amplitude of transmission drops as the metal area increases due to increased reflection. Also, the phase variation is small for $0 \leq a_{1} \leq 4$, however, variation in the phase increases more rapidly for $4 \leq a_{1} \leq 8$. For $a_{1}>7$, even a slight variation in dimensions results into a large variation in the transmission properties.
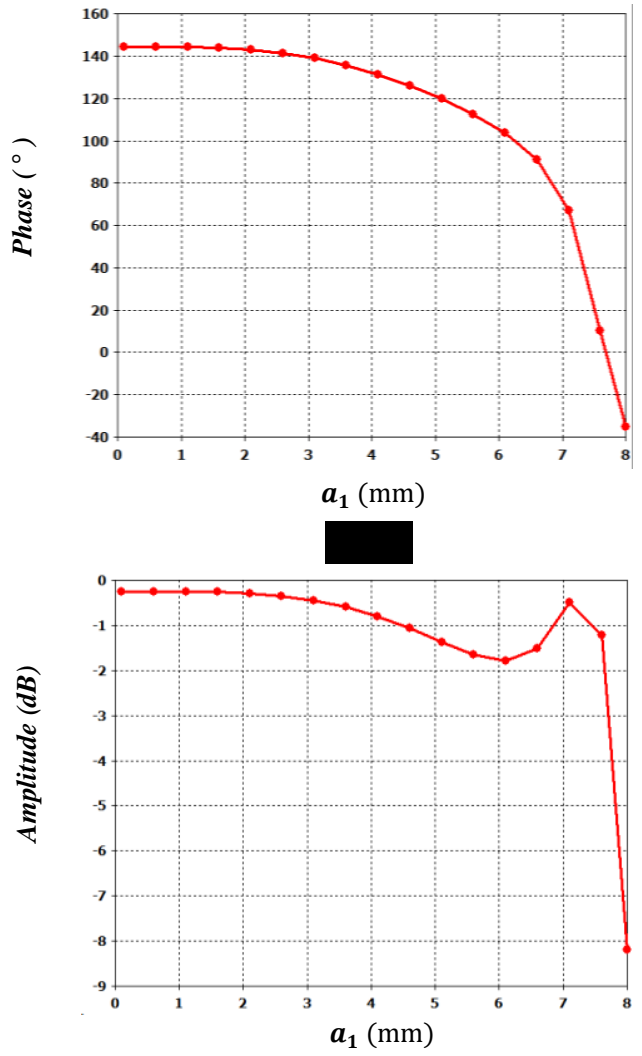

(b)

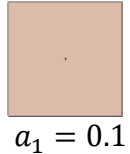

(c)

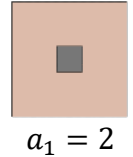

(d)

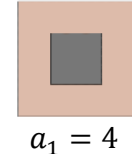

(e)

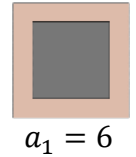

(f)
Figure 27. The effect of variation in the dimensions $\left(a_{1}\right)$ of top and bottom layer. Transmission's (a) phase and (b) amplitude of the cell. The physical appearance of the cell for various values of $a_{1}(c-f)$.

Next, the unit cell was simulated by varying both $a_{1}$ and $a_{2}$ from 0.1 to $8 \mathrm{~mm}$ respectively with a step width of $0.2 \mathrm{~mm}$ due to the available fabrication resolution of $0.2 \mathrm{~mm}$. The transmission properties were recorded for all simulations and the results are plotted in Figure 28 where the transmission phase is represented by independent axis ( $\mathrm{x}$-axis) and the transmission amplitude is represented by $y$-axis. The phases in the plot have been normalised with respect to the phase generated by the cell when $a_{1}=a_{2}=0$, i.e., when the cell is bare of any metal. Thus $0^{\circ}$ in the plot in Figure 28 represents the phase generated by the bare substrate which was found to be $146^{\circ}$. The red line in the plot represents $-3 \mathrm{~dB}$ amplitude while the green line represents $-1 \mathrm{~dB}$ amplitude. Notice that the square unit cell is able to achieve a phase shift range of $0^{\circ}-300^{\circ}$ with amplitude greater than $-3 \mathrm{~dB}$. However, for amplitude greater than $-1 \mathrm{~dB}$ only a limited number of phase shifts can be produced. Also, since the size of the cell is discrete, some phases were not possible to be generated by the cell. However, it is common practice to use approximate phases - when needed - in an application. 


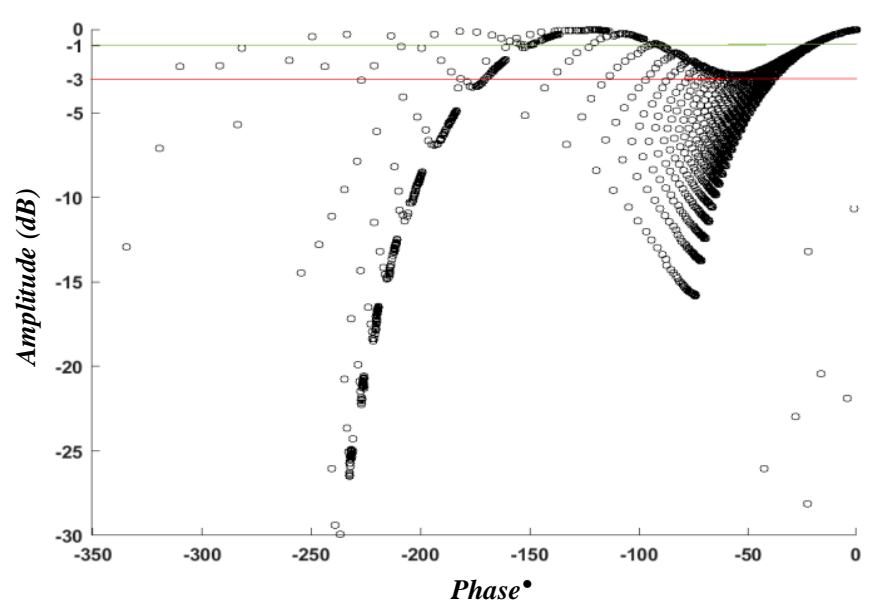

Figure 28. Transmission properties of the square unit cell when its dimensions were varied from 0 to $8 \mathrm{~mm}$.

\section{B. Design of $1 D$ Beam Steering Metasurface}

The first step in the design of an NFSS is to decide on the elevation angle $\theta$ towards which we want to steer the beam of the antenna array. Selection of $\theta$ will greatly affect the design of an NFSS as explained henceforth. The required phase difference $\left(\varphi_{e}\right)$ between adjacent cells of a NFSS to steer the beam towards a particular elevation angle $\theta$ can be calculated using (6). The value of $\theta$ will affect the unit cell design. The number of distinct phases (or equivalently distinct cells) required to produce the desired phase gradient in an NFSS is determined by the value of $\theta$. We choose different values of $\theta$, i.e., $\theta=10^{\circ}, 20^{\circ}, 30^{\circ}, 40^{\circ}, 50^{\circ}$ as an example. Then, according to (6), the corresponding values of $\phi_{e}$ are roughly $20^{\circ}, 40^{\circ}, 60^{\circ}, 80^{\circ}$, and $100^{\circ}$. The NFSS is then designed by combining distinct cells by making sure that a phase difference of $\phi_{e}$ exists between adjacent cells and $\phi_{e}$ progressively increases from left to right (or right to left, depending on the sense of $\theta$, i.e., $+\theta$, or $-\theta$ ) for a complete $360^{\circ}$ cycle. The number of different cells required is equal to the number of distinct phases required to complete a $0-360^{\circ}$ cycle. Also, it is noted that the phase $360^{\circ}$ is equivalent to $0^{\circ}$ and thus a separate cell will not be required for this phase.

The total number of distinct phases required for each $\theta$ selected previously are given in Table 1 where we can see that the number of distinct phases decreases as the value of $\theta$ increases. Also, as pointed out in [21] by the authors, the performance of the metasurface will vary with the value of $\theta$ and is significantly degraded for $\theta>60^{\circ}$. Moreover, for some $\theta$, such as $\theta=40^{\circ}$ and above, the number of distinct phases is not exactly repeatable, and the total number of distinct phases required are truncated to approximately generate complete $360^{\circ}$ cycle. Therefore, to keep the number of distinct phases reasonable and also to keep performance degradation minimum, we choose the value of $\theta$ to be $29^{\circ}$ which approximately corresponds to $\varphi_{e}=60^{\circ}$ and the total number of distinct phases required are thus only $\operatorname{six}\left(\varphi_{e}=\right.$ $0^{\circ}, 60^{\circ}, 120^{\circ}, 180^{\circ}, 240^{\circ}$, and $300^{\circ}$ ) which are also repeatable for phase wrapping.
Table 1. Phase shifts required to design a NFSS for a given $\theta$.

\begin{tabular}{|c|c|l|}
\hline$\theta^{\circ}$ & $\phi_{e}{ }^{\circ}$ & List of distinct pashes required \\
\hline 10 & 20 & $\begin{array}{l}0,20,40,60,80,100,120,140,160,180,200, \\
220,240,260,280,300,320,340 .\end{array}$ \\
\hline 20 & 40 & $0,40,80,120,160,200,240,280,320$. \\
\hline 30 & 60 & $0,60,120,180,240,300$. \\
\hline 40 & 80 & $0,80,160,240,320$, \\
\hline 50 & 100 & $0,100,200,300$ \\
\hline
\end{tabular}

For the construction of the NFSS, the dimensions of the cells corresponding to the values of $\phi_{e}$ are selected from Figure 28. There are several possibilities but we will select phases with highest transmission amplitude to reduce reflection and ohmic losses. These cells are then combined to construct the NFSS. Phases selected for $\theta=29^{\circ}$ are tabulated in Table 2 along with dimensional values for their cells. The constructed NFSS is shown in Figure 29 along with the array. It is a common practice in the research community that after constructing an NFSS, an extensive optimisation is carried out to optimise the steering results, reduce side lobe levels and other properties of an NFSS.

Table 2. Dimensional values of the square cells to construct a NFSS for $\theta=29^{\circ}$.

\begin{tabular}{|c|c|c|c|c|}
\hline $\begin{array}{c}\text { Required } \\
\phi_{e}{ }^{\circ}\end{array}$ & $\begin{array}{c}a_{1} \\
(\mathrm{~mm})\end{array}$ & $\begin{array}{c}a_{2} \\
(\mathrm{~mm})\end{array}$ & $\begin{array}{c}\text { Cell's } \\
\text { normalised } \\
\text { phase }^{\circ}\end{array}$ & $\begin{array}{c}\text { Cell's } \\
\text { amplitude } \\
(\mathrm{dB})\end{array}$ \\
\hline 0 & 0 & 0 & 0 & -0.05 \\
\hline 60 & 7.2 & 6.4 & -59.97 & -0.09 \\
\hline 120 & 8.0 & 6.9 & -120.12 & -0.41 \\
\hline 180 & 8.1 & 8.6 & -182.23 & -0.98 \\
\hline 240 & 8.4 & 8.5 & -243.60 & -1.01 \\
\hline 300 & 8.4 & 8.6 & -310.04 & -1.22 \\
\hline
\end{tabular}

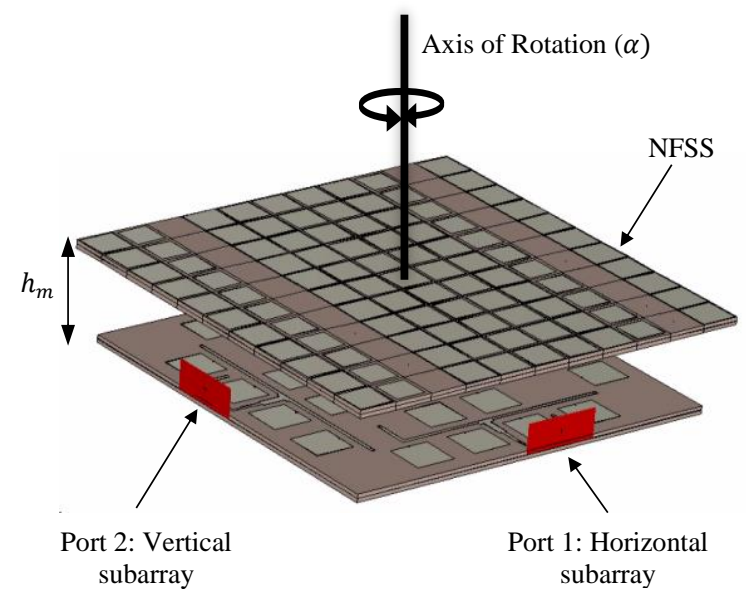

Figure 29. Constructed NFSS placed on top of the array.

The NFSS is placed on top of the array at a distance $h_{m}$. This distance can be varied to obtain optimum results. Usually values of $h_{m}$ is selected to be greater than $\lambda_{0} / 2$ to avoid 
operation in the reactive near field region where EM interaction is unknown. Moreover, if the NFSS is too close to the array surface it will result into strong coupling and reflections which will alter the EM properties of the array.

It is important to mention here that it is a common practice in research community that a base antenna or array is usually accompanied by a phase correction surface (PCS) which smooths out the phase profile of the base antenna so that it has less phase variation [22]. This is done to comply with the assumption made during the design of a unit cell where the input wave is assumed to be a plane wave. The phase of the incoming wave for all instances of the unit cell is considered to be constantly zero. In reality, this is not true as the phase of the near-field of a base antenna varies along the aperture of the array. However, a PCS is an additional piece of hardware that not only contribute to the cost of the whole antenna system but also to its overall profile. Therefore, a PCS is used only when there is a large variation in the phase profile of the base antenna or array such as in the case of a dielectric resonator antenna (DRA) [22]. The phase profile of the dual polarised array presented in Section 3 is shown in Figure 30. A twodimensional plane cut shows the surface phase profile of the array when one sub-array is excited at a time while a onedimensional phase profile is also given along the centre of the array for each sub-array. Note that the phase of the array is quite homogeneous along the sub-array's axis (except at the edges due to discontinuity). Since the variation in the phase of the dual polarised array is not that significant, a PCS will not be required.

The fabricated model and measurement setup for one- and two-dimensional (2D) beam steering is shown in Figure 31 (a) and (b) respectively. In this subsection we will present $1 \mathrm{D}$ steering results from simulation and measurement. The steering will be performed for linear and circular polarisations. A full 2D steering with a scaled version of the DP array will be presented in the next subsection.
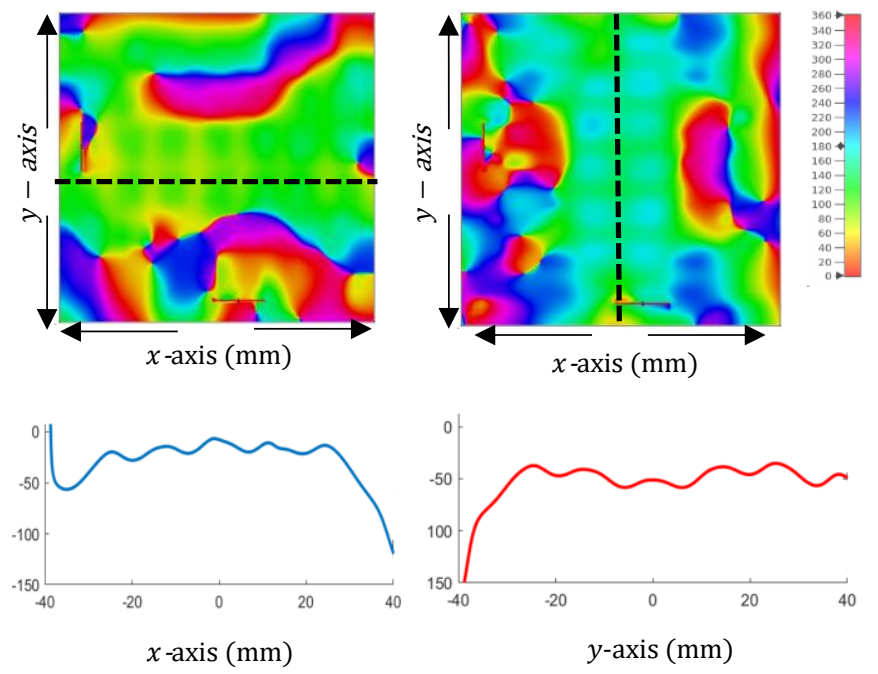

(a)

(b)

Figure 30. Phase profile of the dual polarised array. The phase profile of (a) horizontal sub-array and (b) vertical sub-array. The colour $2 D$ plot shows the surface phase profile of array while line plots show the phase along the indicated central lines.

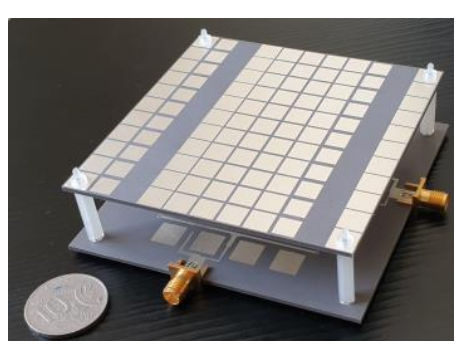

(a)

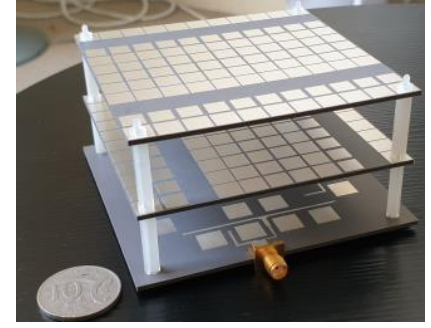

(b)
Figure 31. Fabricated NFSS; (a) one NFSS for 1D steering, (b) two NFSS setup for $2 D$ steering. An Australian 10 cent coin is also shown for relative size.

We simulated the array with the designed NFSS for the selected $\theta$ for various settings. The results for horizontal subarray are given in Figure 32 for the three azimuth angles, i.e., $\phi=0^{\circ}, 45^{\circ}, 90^{\circ}$ when the beam was steered in those directions. Solid curve in Figure 32 represents results from simulation whilst the dashed curve are the steering results from measurement. During this operation the vertical subarray was kept inactive. The results for vertical subarray would be similar due to reciprocity. Looking at Figure 32, the steering results are quite accurate within $1.3^{\circ}$ error range. Though, the measured patterns show a drop of about $1.2 \mathrm{~dB}$ in the peak gain when compared to the simulation results. This could be due to nonideal conditions in the measurement setup or the quality of the fabricated prototypes. Moreover, during the simulations conducted in CST Microwave Studio we excited the array with an ideal waveport, however, during measurement the array was excited by feeding its SMA connectors with external input coming from the anechoic chamber. This difference in excitation conditions could also contribute to the minor loss in the peak directivity.

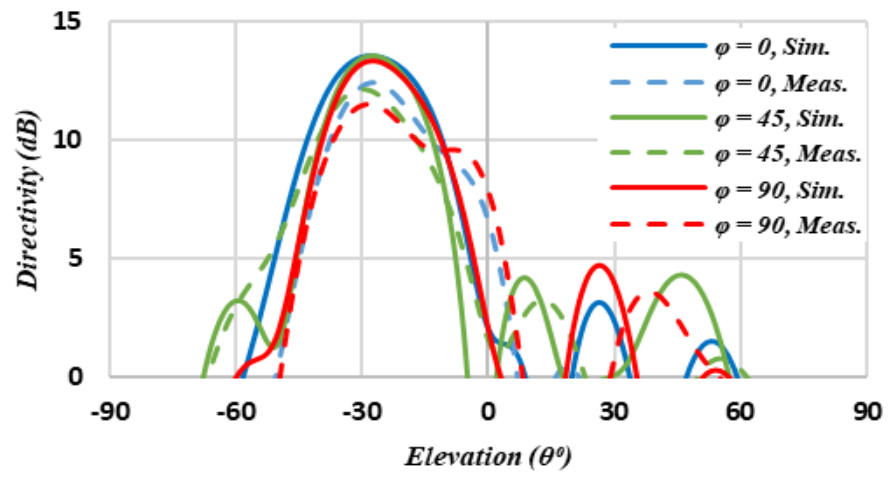

Figure 32. One-dimensional steering towards $\theta=29^{\circ}$ with an NFSS.

Simulation and measurement were also conducted for steering in the LHCP mode with both subarrays switched on and appropriate phase difference in their inputs. Their steering results are shown in Figure 33. For coherency, similar directions were chosen for steering as that for the LP case. Here, the beam is narrower compared to the LP case as both subarrays 
are operational making the beam narrow in all directions. Also, the peak directivity level is higher than the LP case. However, it comes at a cost of higher sidelobe level which was expected.

The antenna array that we used so far for 1D steering is a medium gain array. Medium gain antennas are not suitable for two-dimensional (2D) steering as the inherent sidelobe level introduced by a pair of NFSS [23] and greater attenuation in peak directivity by two NFSS are not desirable. To better analyse the performance of the shared-aperture approach for 2D beam steering we will consider a large number of elements for the array in order to get high gains and narrow beam shape for several azimuth angles. This will be discussed in next section for two-dimensional beam steering.

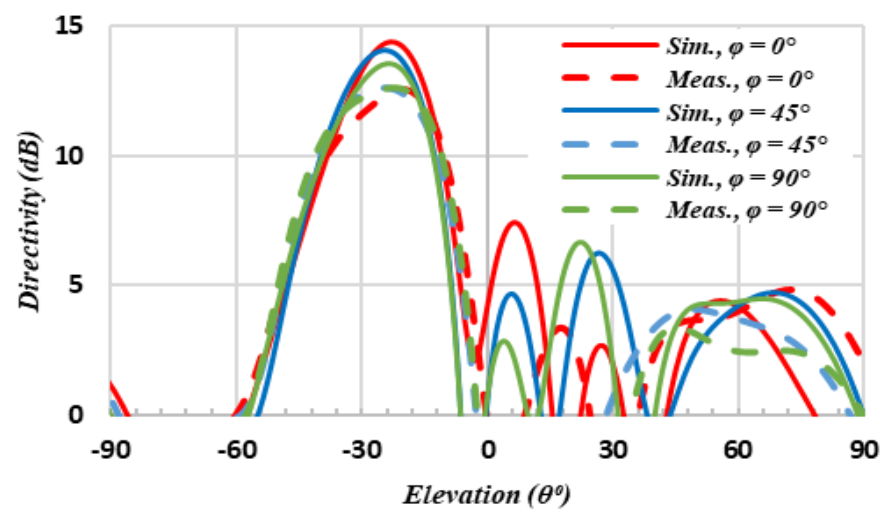

Figure 33. One-dimensional steering when array steered towards $\theta=$ $29^{\circ}$ in LHCP mode.

\section{2 D Beam Steering}

Figure 24 shows the scaled version of the shared-aperture array presented in Section II. The array has 32 elements in each subarray thus the total size of the array is $32 \times 32$ minus the empty diagonal slots within the aperture. The beam patterns of this array were already presented in Figure 25 where we see the beam is very similar in both orthogonal planes. This high gain is desirable for two-dimensional beam steering as the use of two NFSSs simultaneously attenuates the beam of the array and induces high sidelobe levels.

Figure 34 shows the concept for 2D beam steering where we see that a pair of NFSSs are used to steer the beam in elevation and azimuth. The angle of rotation of the first NFSS and the second NFSS is denoted by $\psi_{1}$ and $\psi_{2}$ respectively. $\delta_{1}$ and $\delta_{2}$ are the elevation angle of the beam steered by first NFSS and second NFSS respectively. The final direction of the beam is denoted by $\theta$ and $\phi$. The detailed formulation and mechanism of metasurface based beam steering are elaborated in [24] [25]. Reference [24] uses the concept of vector summation or paraxial approximation method focusing mainly on the direction of waves after passing through each metasurface. While reference [19] uses the concept of phase delay that is generated in the incident waves by each NFSS. Due to several ideal assumptions, paraxial approximation method is less accurate, specifically for near-field steering. This is further explained in Figure 35 where the estimated beam path of the resultant beam after passing through both metasurfaces is shown.

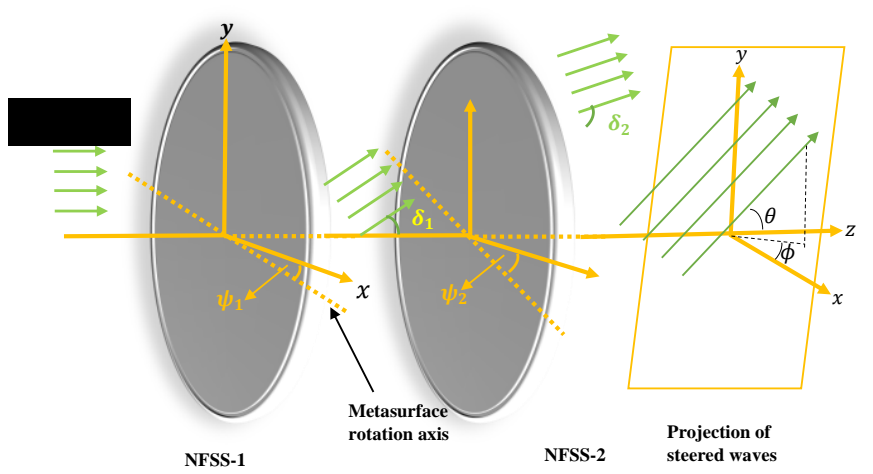

Figure 34. Two-dimensional beam steering using near-field metasurfaces

The estimate of the elevation and azimuth angles $(\theta, \phi)$ of the steered beam using the paraxial approximation method [24] is given by:

$$
\begin{gathered}
\theta=\sqrt{\delta_{1}^{2}+\delta_{2}^{2}+2 \delta_{1} \delta_{2} \cos \left(\psi_{1}-\psi_{2}\right)} \\
\phi=\tan ^{-1} \frac{\left|\delta_{1} \sin \psi_{1}+\delta_{2} \sin \psi_{2}\right|}{\left|\delta_{1} \cos \psi_{1}+\delta_{2} \cos \psi_{2}\right|}
\end{gathered}
$$

Whereas the estimate of the elevation and azimuth angles $(\theta, \phi)$ of the steered beam using the phase method [19] is given by:

$$
\begin{gathered}
\theta=\sin ^{-1}\left(\frac{\sqrt{p_{1}^{2}+p_{2}^{2}+2 p_{1} p_{2} \cos \left(\psi_{1}-\psi_{2}\right)}}{k}\right) \\
\phi=\tan ^{-1}\left(\frac{p_{1} \sin \psi_{1}+p_{2} \sin \psi_{2}}{p_{1} \cos \psi_{1}+p_{2} \cos \psi_{2}}\right)
\end{gathered}
$$

where $p_{1}, p_{2}$ (expressed in $\mathrm{rad} / \mathrm{m}$ ) are the surface phase gradient of NFSS- 1 and NFSS- 2 respectively. The inverse problem, i.e., the calculation of the rotation angles $\psi_{1}, \psi_{2}$ of the NFSS- 1 and NFSS-2 required to steer the beam towards a particular $(\theta, \phi)$ is given by:

$$
\begin{aligned}
& \psi_{1}=\phi+\frac{1}{2} \cos ^{-1}\left[\frac{(k \sin \theta)^{2}}{2 p^{2}}-1\right] \\
& \psi_{2}=\phi-\frac{1}{2} \cos ^{-1}\left[\frac{(k \sin \theta)^{2}}{2 p^{2}}-1\right]
\end{aligned}
$$

Equation (12) and (13) gives us a rough estimate of the initial positions of the NFSS-1 and NFSS-2 for the desired beam direction $(\theta, \phi)$. However, as previously mentioned, the final beam direction depends on several factors such as accuracy of the phase delay profiles of each NFSS, mutual coupling between the two NFSS and the base array, phase profile of the incident wave to name a few. 


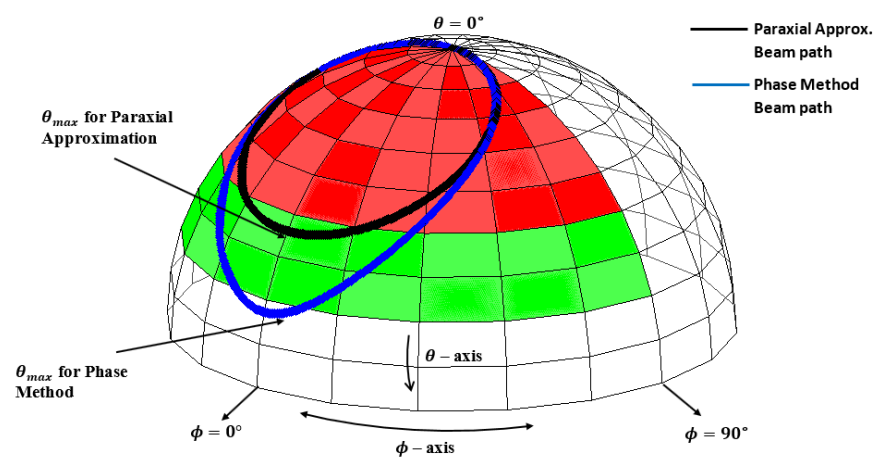

Figure 35. The direction of the steered beam when NFSS-1 is stationary whilst NFSS-2 is rotated from 0 to $\pm \pi$ with $\delta_{1}=28.8^{\circ}$ or $p=116 \mathrm{rad} / \mathrm{m}$.

Notice how the beam path estimated by the phase method shown in Figure 35 has larger maximum elevation angle $\theta_{\max }$ than the one estimated by paraxial approximation method. This difference in value of $\theta_{\max }$ between the two methods increases with the increase in the gradient $(p)$ of the NFSS, i.e., the larger the deflection required from each NFSS the larger the difference between the estimate produced by each method. This is because paraxial approximation method assumes smaller elevation angles in its formulation. Therefore, we will use the more accurate phase method for our steering results. It is important to note that both the paraxial approximation and phase methods assumes ideal conditions such as plane wave input, no mutual coupling or reflection etc. and therefore, their estimate is only an approximation of beam steering results. The results obtained from simulation and measurement represents more realistic estimate of the beam steering system.

Now we will consider several scenarios for beam steering and present corresponding simulation results. We assume that both NFSS- $1 \&-2$ have the same phase gradient $p$ (which also means $\delta_{1}=\delta_{2}=\delta$ ). There are several cases for NFSS rotations which produce different steering of the beam. These scenarios are briefly explained below:

1. Keeping NFSS-1 stationary while rotating NFSS-2: In this case, the azimuth angle of the steered beam will directly correspond to the rotation of the NFSS- 2 by a factor of half while the elevation angle will start from the maximum value (greater than $2 \delta$, exact value depends on value of $p$, the larger the value of $p$ the larger the maximum achievable elevation angle) at $\psi_{2}=0$ and reach towards broadside $(\theta=0)$ as we keep rotating NFSS-2 up to $\psi_{2}= \pm 180^{\circ}$.

2. First rotating NFSS-2 to direct the beam towards a specific $\theta$ and then co-rotating NFSS- 1 and NFSS- 2 to rotate the beam in a cone of constant $\theta$ towards any desired $\phi$.

3. To steer the beam along the elevation while keeping the azimuth angle constant, the two NFSS are rotated in opposite direction with equal rate of rotation. If we select the deflection of both NFSS to be $\delta=30^{\circ}$ or $p=120$ $\mathrm{rad} / \mathrm{m}$, then the full range of elevation $0^{\circ} \leq \theta \leq 90^{\circ}$ can be achieved according to the Phase Method [19].

Due to limited space and large times taken by the simulation of complex structure of two NFSS placed on top of a $32 \times 32$ version of the shared-aperture DP array presented in Section II, we will limit our steering to few positions. Moreover, we will consider the steering of the DP array for one case only for each of linear and circular polarisation.

We choose a set of directions towards which we want to steer the beam of the array which is given in Table 3 . The values in Table 3 have been calculated using (12) and (13). The steering involves two sets of rotations. First, to steer the beam towards a particular $\theta$, NFSS- 1 is kept stationary at $\psi_{1}=0^{\circ}$ while NFSS-2 is rotated towards $\psi_{2}$ for which values are given in Table 3, column 3. Then, to steer the beam along a cone of aforementioned $\theta$ towards a particular $\phi$ both NFSS- $1 \& 2$ are co-rotated by an angle given in column 4 of Table 3 .

Table 3. Rotation angles of NFSS-1 \& 2 for various beam positions with $\delta=29^{\circ}$ or $p=116 \mathrm{rad} / \mathrm{m}$.

\begin{tabular}{cccc}
\hline \hline $\begin{array}{c}\text { Azimuth } \\
\left(\phi^{\circ}\right)\end{array}$ & $\begin{array}{c}\text { Elevation } \\
\left(\theta^{\circ}\right)\end{array}$ & $\begin{array}{c}1^{\text {st }} \text { rotation: } \\
\text { NFSS-2 only } \\
\left(\psi_{2}^{\circ}\right)\end{array}$ & $\begin{array}{c}2^{\text {nd }} \text { rotation: } \\
\text { Co-rotation of } \\
\text { NFSS-1 \& 2 } \\
\left(\psi_{1}=\psi_{2}{ }^{\circ}\right)\end{array}$ \\
\hline 0 & 20 & 138.5 & -69.2 \\
20 & 30 & 117.5 & -38.7 \\
60 & 45 & 85.8 & 17.1 \\
40 & 60 & 52.5 & 13.8 \\
\hline
\end{tabular}

Now we will present simulation results for LHCP beam steering by using array in LHCP mode with two NFSSs. It is assumed steering results will be similar for RHCP due to reciprocity and will not be repeated. The radiation patterns of the output wave when steered with a pair of NFSS's is given in Figure 37 where we see that the steering was successfully achieved with minor error. As the steering elevation angle increases the beam widens and the directivity drops. This behaviour is typical of any beam steering. Also, the error in the final steered position increases we as increase the desired elevation angle.

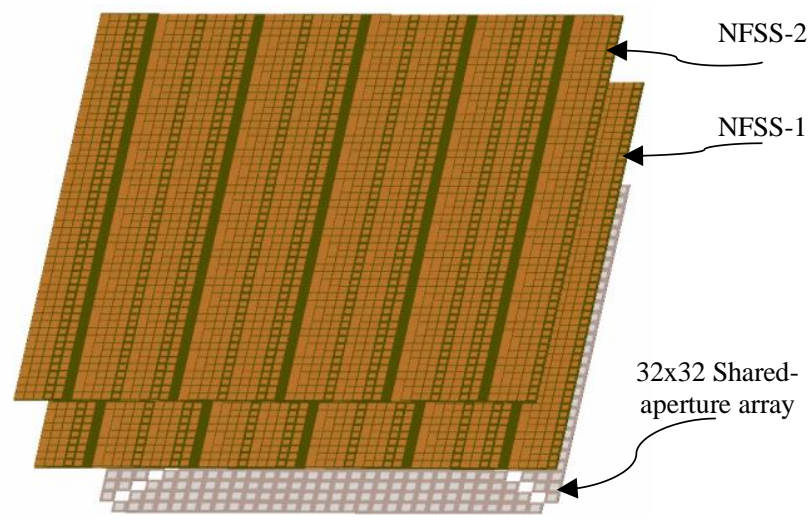

Figure 36. Setup for $2 D$ beam steering. 


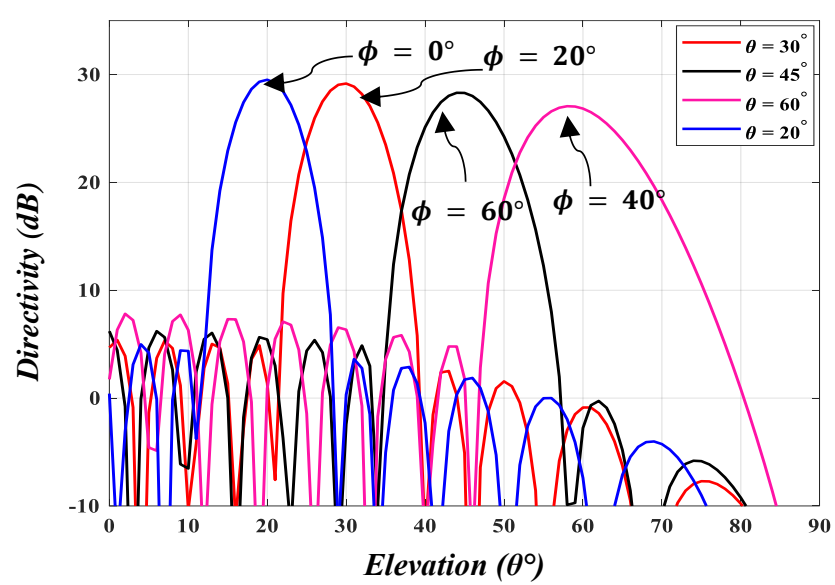

Figure 37. Radiation patterns of the output wave when steered with a pair of NFSS's in the directions given in Table 3.

\section{CONCLUSION}

A polarisation-reconfigurable shared aperture array has been presented in this paper. The shared aperture approach makes it possible to avoid the use of active switches such as pin diodes within the array aperture which eliminates the insertion loss of active components. Moreover, the array was shown to be capable of producing all linear and circular polarisation radiation. The level of gain, directivity, and side lobes were found to be in good range, based on the results obtained from simulation and measurement. A good cross-pol separation as well as port isolation were observed in the simulation results of the array. The array was further tested for 1D and 2D beam near-field beam steering for various polarisations. The beam steering results were observed to be accurate within $1.3^{\circ}$ while $2 \mathrm{D}$ beam steering was achiaeved within $1^{\circ}-2.3^{\circ}$ error range.

\section{REFERENCES}

[1] D. D. Grieg and H. F. Engelmann, "Microstrip-A New Transmission Technique for the Klilomegacycle Range," in Proceedings of the IRE, 1952.

[2] J. Li, J. Shi, K. Xu, Q. Yang, J. Chen and A. Zhang, "Polarization reconfigurable annular ring slot antenna design," AEU - International Journal of Electronics and Communications, vol. 123, 2020.

[3] L.S.Yang, L. Yang, Y. A. Zhu, K. Yoshitomi and H. Kanaya, "Polarization reconfigurable slot antenna for $5.8 \mathrm{GHz}$ wireless applications," AEU - International Journal of Electronics and Communications, vol. 101, pp. 27-32, 2019.

[4] R. L. Haupt and M. Lanagan, "Reconfigurable Antennas," IEEE Antennas and Propagation Magazine, vol. 55, no. 1, pp. 49-61, 2013.

[5] H. Sun and S. Sun, "A Novel Reconfigurable Feeding Network for Quad-Polarization-Agile Antenna Design," IEEE Transaction on Antennas and Propagation, vol. 64, no. 1, pp. 311-316, 2016.
[6] S. Lee and Y. Sung, "Simple polarization-reconfigurable antenna with t-shaped feed," IEEE Antennas and Wireless Propagation Letters, vol. 5, pp. 114-117, 2016.

[7] P. Qin, A. Weily, Y. Guo and C. Liang, "Polarization reconfigurable u-slot patch antenna," IEEE Transactions on Antennas and Propagation, vol. 58, no. 10, pp. 33833388, 2010.

[8] H. Zhu, S. Cheung, X. Liu and T. Yuk, "Design of polarization reconfigurable antenna using metasurface," IEEE Transactions on Antennas and Propagation, vol. 62, no. 6, pp. 2891-2898, 2014.

[9] K. Kandasamy, B. Majumder, J. Mukherjee and K. P. Ray, "Low-RCS and Polarization-Reconfigurable Antenna Using Cross-Slot-Based Metasurface," IEEE Antennas and Wireless Propagation Letters, vol. 14, pp. 1638-1641, 2015.

[10] Z. Wu, H. Liu and L. Li, "Polarization reconfigurable metasurface superstrate antenna with low profile," in 10th European Conference on Antennas and Propagation (EuCAP), Davos, 2016.

[11] D. M. Pozar and S. D. Targonski, "A shared-aperture dual-band dual-polarized microstrip array," IEEE Transactions on Antennas and Propagation, vol. 49, no. 2, pp. 150-157, 2001.

[12] J. Zhang, W. Wu and D. Fang, "Dual-Band and DualCircularly Polarized Shared-Aperture Array Antennas With Single-Layer Substrate," IEEE Transactions on Antennas and Propagation, vol. 64, no. 1, pp. 109-116, 2016.

[13] F. Q. e. al., "A Simple Low-Cost Shared-Aperture DualBand Dual-Polarized High-Gain Antenna for Synthetic Aperture Radars," IEEE Transactions on Antennas and Propagation, vol. 64, no. 7, pp. 2914-2922, 2016.

[14] C. Mao, S. Gao, Q. Luo, T. Rommel and Q. Chu, "LowCost X/Ku/Ka-Band Dual-Polarized Array With Shared Aperture," IEEE Transactions on Antennas and Propagation, vol. 65, no. 7, pp. 3520-3527, 2017.

[15] S. O. Kasap, Optoelectronics and Photonics: Principles and Practices, New Jersey: Prentice-Hall, 2001.

[16] T. Taylor, ““'Design of Line-Source Antennas for Narrow Beamwidth and Low Side Lobes," Transactions of the IRE Professional Group on Antennas and Propagation, vol. 3, no. 1, pp. 16-28, 1955.

[17] H. Ali, M. U. Afzal, K. P. Esselle and R. M. Hashmi, "Intergration of Geometrically Different Elements to Design Thin Near-Field Metasurfaces," IEEE Access, vol. 8, pp. 225336-225346, 2020.

[18] N. Gagnon and A. Petosa, "Using Rotatable Planar Phase Shifting Surfaces to Steer a High-Gain Beam," IEEE Transactions on Antennas and Propagation, vol. 61, no. 6, pp. 3086-3092, 2013.

[19] X. Zhao, C. Yuan, L. Liu, S. Peng, Q. Zhang, L. Yu and e. al, "All-Metal Beam Steering Lens Antenna for High Power Microwave Applications," IEEE Transaction on Antenna and Propagation, vol. 65, no. 12, pp. 73407344, 2017. 
[20] A. A. Baba, R. M. Hashmi, K. P. Esselle, M. Attygalle and D. Borg, "A Millimeter-Wave Antenna System for Wideband 2-D Beam Steering," IEEE Transactions on Antenna and Propagation, vol. 68, no. 5, pp. 3453-3464, 2020.

[21] H. Taghvaee, S. Abadal, A. Pitilakis, O. Tsilipakos, A. C. Tasolamprou and C. Liaskos, "Scalability analysis of programmable metasurfaces for beam steering," IEEE Access, vol. 8, p. 105320-105334, 2020.

[22] M. U. Afzal, A. Lalbakhsh and K. P. Esselle, "Method to Enhance Directional Propagation of Circularly polarized Antennas by Making Near-Electric Field Phase More Uniform," IEEE Transaction on Antennas and Propagation, vol. 69, no. 8, pp. 4447-4456, 2021.

[23] K. Singh, M. U. Afzal, M. Kovaleva and K. P. Esselle, "Controlling the Most Significant Grating Lobes in Two-Dimensional Beam-Steering Systems With PhaseGradient Metasurfaces," IEEE Transactions on Antennas and Propagation, vol. 68, no. 3, pp. 13891401, 2020.

[24] C. D. McEwen and M. R. Khan, "Beam Steering Method with Improved Sidelobe Response using Dielectric Wedges for Satellite TV Reception," in 14th European Microwave Conference, 1984.

[25] J. Wang and Y. Rahmat-Samii, "Phase Method: A More Precise Beam Steering Model for Phase-Delay Metasurface Based Risley Antenna," in 2019 URSI International Symposium on Electromagnetic Theory (EMTS), 2019. 